\title{
High intensity versus low intensity family-based childhood obesity treatment programs: Effectiveness, engagement and implementation outcomes from a randomized controlled trial in medically underserved region
}

Jamie Zoellner ( $\sim$ jz9q@virginia.edu )

University of Virginia https://orcid.org/0000-0001-5445-5594

Wen You

University of Virginia

Jennie Hill

University of Nebraska Medical Center

Donna-Jean Brock

University of Virginia

Maryam Yuhas

University of Virginia Department of Public Health Sciences

Bryan Price

University of Virginia

Jonathon Wilson

City of Danville, Parks and Recreation

Danielle Renee Montague

City of Danville, Parks and Recreation

Paul Estabrooks

University of Nebraska Medical Center

\section{Research}

Keywords: childhood obesity, behavioral intervention, comparative effectiveness research, implementation science, community-based participatory research, medically underserved area, minority groups

Posted Date: June 18th, 2020

DOI: https://doi.org/10.21203/rs.3.rs-35413/v1

License: (c) (i) This work is licensed under a Creative Commons Attribution 4.0 International License. Read Full License 


\section{Abstract \\ Background}

Access to evidence- and family-based childhood obesity (FBCO) treatment interventions in the US is a challenge, especially in medically underserved regions where disparities in childhood obesity persist.

\section{Methods}

A comparative effectiveness RCT of two 6-month FBCO treatment interventions varied in structure and contact hoursiChoose (high intensity) and Family Connections (low intensity)-in a medically underserved region. Guided by a systems-based and community-based participatory research approach and the RE-AIM planning and evaluation framework, this paper reports on effectiveness (BMI z-scores) and implementation outcomes. Changes in parent outcomes, secondary outcomes (e.g. QOL, nutrition, physical activity), engagement, and implementation costs are also reported. Analyses included descriptive statistics and intention-to-treat Heckman treatment effect models with cluster robust inference adjustment.

\section{Results}

Enrolled children ( $n=139$, mean age $10.1 \pm 1.7$ years, $30 \%$ overweight, $70 \%$ obese, $45 \%$ black, $63 \%$ on Medicaid) were randomly assigned to iChoose $(n=70)$ or Family Connections $(n=69)$. Retention rates were $63 \%$ for $i$ Choose and $84 \%$ for Family Connection. Among children, 6-month BMI z-score changes were not statistically significant within iChoose [BMI z-score $0.03(95 \% \mathrm{Cl}=-0.13,0.19)$ ] or Family Connections [BMI z-score $0.00(95 \% \mathrm{Cl}=-0.16,0.16)$ ]. Likewise, relative between condition effects were not statistically significant and similar null effects were found in parents' BMI changes. Yet relative to iChoose, Family Connections parents had significant improvement in quality of life $(p=0.03)$. Both programs were delivered with high fidelity (77-100\%). iChoose engagement was $30 \%$ family classes, $36 \%$ IVR calls, and $25 \%$ physical activity classes; Family Connections engagement was $52 \%$ parent classes and $61 \%$ IVR calls. Implementations costs per children with improved BMI z-score was \$2,841 for iChoose and \$955 for Family Connections.

\section{Conclusions}

Both FBCO treatment interventions were delivered with high fidelity; yet neither yielded significant improvements in child or parent BMI. Relative to iChoose, descriptive data indicated higher retention, better engagement, and lower costs for Family Connections-suggesting that a lower intensity program may better fit the intended audience's context. Future research efforts need to address lack of program effects and further explore strategies to leverage higher engagement in lower intensity programs for populations in medically underserved areas.

\section{Trial registration:}

Clincialtrials.gov: NCT03245775. Registered 10 August 2017. https://clinicaltrials.gov/ct2/show/NCT03245775? term $=$ NCT03245775\&draw $=2 \&$ rank $=1$

\section{Background}


Approximately one in three children in the United Sates (US) are overweight, and about one in five children are obese [1]. Also alarming, are persistent racial/ethnic and socioeconomic disparities for childhood obesity, as numerous studies have shown unequal burden on minority, low income, and rural children [2-4]. Reflective of the prevalence, disparities, and associated health consequences, childhood obesity is a national priority $[1,5,6]$. Unfortunately, access to evidencebased childhood obesity interventions in the US is a challenge, especially in medical underserved regions [7].

There is a large body of empirical work documenting the efficacy of family-based childhood obesity (FBCO) treatment interventions [8-21]. Similarly, evidence summaries from the US Preventive Services Task Force (USPSTF) highlight the efficacy of family-centered, comprehensive intensive, behavioral, interventions for childhood obesity treatment [22]. In brief, accumulated evidence indicates that 26 contact hours or more and over a 2-12 month time frame result in child weight loss, and multicomponent interventions with 52 or more contact hours demonstrate greater weight loss and some improvements in metabolic risk factors [22]. Despite demonstrated efficacy by these high contact hour treatment interventions, the ability for families to adhere to high intensity programs, as well as capacity for local health and community organizations to adopt and sustain these programs, is less clear.

It is imperative to understand adherence and attrition in FBCO treatment programs. In general, higher adherence is associated with a higher likelihood of improved weight outcomes [22]. However, paradoxically these higher intensity programs often have a lower likelihood of participant adherence [23-29]. This highlights the need for heightened efforts to better understand potential impacts and family burden of FBCO treatment interventions of varying intensity (e.g. number of contact hours, number and type of intervention sessions, program duration).

The intensity and structure of FBCO treatment programs also influences the ability of local community and clinical systems to adopt and sustain interventions [30]. Key factors that impact organizational-level adoption and maintenance include expertise of intervention delivery staff, facility and resource demands, and overall intervention delivery costs. In health disparate regions, where childhood obesity is typically disproportionately high and evidence-based FBCO treatment options are often unavailable, understanding the appeal and practicality of interventions from the organizational-level perspective is vital [30].

In light of these documented challenges and opportunities, there is a clear need to compare interventions that meet the USPSTF recommended number of contact hours to interventions of lower intensity contact hours within the context of a health disparate region [31]. Of further need is that program design, implementation and evaluation approaches consider both individual-level and organizational-level perspectives. The current study builds from a 10-year community-academic partnership in the Dan River Region of Virginia and a FBCO treatment pilot trial [30, 32-34]. It was designed to address identified gaps in the literature and targeted community. Focused on this health disparate region, this research compares two evidence-based FBCO treatment programs (iChoose vs. Family Connections) that vary in contact hours, structure, and implementation demands [13, 17-20, 35].

This project was guided by a systems-based and community-based participatory research (CBPR) approach and the REAIM planning and evaluation framework [36-42]. The main focus of this manuscript is on individual-level effectiveness outcomes among both children and parents enrolled the randomized controlled trial (RCT) of two FBCO treatment interventions. More specifically, the primary aim of this manuscript is to examine 6-month changes in child BMI z-score, by randomized intervention condition. Secondary aims are to determine 6-month intervention effects on parent BMI level and additional child and parent anthropometric, blood pressure, and self-reported outcomes (i.e., quality of life, nutrition, physical activity). Implementation outcomes were also assessed in terms of fidelity to protocol and organizational-level program implementation costs. Differences in program engagement, retention, and costs per child participant with improved BMI z-scores were also examined. 


\section{Methods}

\section{Study design}

This comparative effective research (CER) study included an RCT of two FBCO treatment interventions that varied in structure and number of contact hours, including iChoose (high intensity) and Family Connections (low intensity). This study was powered to test the hypothesis that children randomized into iChoose would achieve significantly greater improvements in BMI z-scores as compared to children randomized to Family Connections [43].

Three cohorts of eligible families (children and parents) were enrolled and randomized in Fall 2017, Spring 2018, Spring 2019. The University of Virginia's Institutional Review Board approved this research. Parents provided written informed consent and children provided assent at enrollment. To compensate time involved in data collection efforts, both parents and children received gift cards including \$25 at baseline, \$25 at 3-month, and \$50 at 6-month assessments. Full study details are in the published protocol paper [43].

\section{Study setting and context}

This RCT targeted the city of Danville and surrounding Pittsylvania county, which is located in the Dan River Region of south central Virginia. This federally designated, medically underserved area is characterized by a small city that serves as a regional hub for numerous surrounding large rural counties. The region is a convergence of social determinants of health that includes low socio-economic status, high rates of unemployment, and high proportion of racial minorities [44-47]. According to the Virginia 2020 County Health Rankings for health factors, the city of Danville and Pittsylvania county rank 127 and 97, respectively, out of 133. These ratings reflect regional disparities for health behaviors, clinic care, social and economic, and physical environment factors. Although data are limited, one local school district reveal childhood obesity rates three times higher than state averages [48].

The regional childhood obesity Community Advisory Board (CAB) and CBPR partnership leading this research originated in January 2013. In the initial phase, CAB goals were to advance regional community capacity to develop and implement a FBCO treatment intervention and to pilot test the intervention [30, 33, 34, 49]. Transitioning into the current RCT, CAB partners continued involvement in all aspects of the research. Primary roles of clinical partners, Sovah Pediatrics and Piedmont Access to Health Services, Inc. (PATHS; a Federally Qualified Health Center), included family recruitment and referral. These two local clinical systems represent the largest pediatric providers in the region. Whereas the City of Danville Parks \& Rec led all program delivery and implementation efforts, including program engagement and retention efforts, and assisted with recruitment efforts. A parent advisory team (PAT), consisting of iChoose graduates from the initial pilot trial, also assisted with this RCT [50]. Adapted from community health worker models [51-53], main roles of the PAT were to optimize program recruitment, engagement, and study retention by providing a social network for program families. The interdisciplinary team of academic research partners helped organize CAB and PAT functions and provided oversight for study related training, technical assistance, and data collection activities. Additional details of the CAB and PAT formation and capacity advancement have been previously published [33, 34, 43, 50].

\section{Eligibility, recruitment, and randomization}

To be eligible families must have resided in the Dan River Region, be English speaking, have a child with a BMI percentile ranking $\geq 85 \%$, and at least one parent or caregiver (hereafter referred to as parents) willing to participate in the program. For Cohorts 1 and 2, eligibility criteria for age was 8- to 12-year-old children. For Cohort 3, the age criterion was extended to 5- to 12-year-old children to meet trial recruitment needs. Families were excluded if their child had a major cognitive impairment. 
Two main recruitment strategies, including medical chart review and referral by two clinical partners and an open referral system, are detailed in Fig. 1. In total there were 1,802 referrals (Fig. 2, CONSORT). Of 1,802 referrals 1,783 (99\%) were physician referrals and 19 (1\%) self-referral from open enrollment efforts. Also, 881 (49\%) referrals were screened via telephone and 921 (51\%) were not screened due to a non-functioning number or expended call attempts. Of those screened, 790 (90\%) were eligible and 226 (29\%) scheduled an enrollment appointment. Figure 2 describes reasons for ineligibility and decline. Of those who scheduled an enrollment appointment, 139 (62\%) enrolled in the study. The overall enrollment rate was $8 \%$ of total referrals $(n=1,802)$ and $18 \%$ of those contacted and determined eligible during the screening process $(n=790)$.

Within each cohort, randomization occurred at the family level. Families selected their randomized assignment from concealed envelopes that resulted in 70 families randomized to iChoose and 69 families to Family Connections. For iChoose families, completion of 3-month and 6-month follow-up assessments were $66 \%$ and $63 \%$, respectively. For Family Connection families, completion of 3-month and 6-month follow-up assessments were $77 \%$ and $84 \%$, respectively.

\section{Family-based childhood obesity (FBCO) treatment intervention descriptions and strategies for implementation, engagement and retention}

Figure 1 illustrates the underlying conceptual frameworks and key 6-month intervention components. iChoose is adapted from an established evidence-based FBCO, Bright Bodies,[18-20, 30, 49] targets the parent/child dyad, and includes approximately 64 intended contact hours. Family Connections[35] is an adaptation of Golan's Home Environmental Change model [17], focuses exclusively on parents as agents of change, and includes about 5 intended contacted hours. Figure 1 also details study protocols for the consultee-centered program training approach for Parks \& Recreation staff $[30,54]$, structured fidelity assessments, and comprehensive engagement and retention strategies.

\section{Measures}

Individual-level outcome measures. At the initial telephone screening, demographic information was collected [55-57]. To allow for reach and representativeness analysis, collection of demographic information was also attempted among those who were eligible but declined to participate [58].

Data collection occurred at a centrally located Parks \& Rec site. In-person measures of height, weight, blood pressure and waist circumference were completed by staff using research grade equipment and published guidelines [59, 60]. A computer-assisted interview program, developed for this project, administered surveys to participants. Previously validated instruments were used to evaluate parent health literacy at baseline [61] and to assess all secondary outcomes among children and parents, including quality of life (QOL) [62, 63], nutrition [64-67], and physical activity [68]. For children, self-reported measures were only collected among those age eight years and older; except for the QOL measure which was administered to all children regardless of age. Baseline and 6-months assessments included all described outcomes, while the 3-month assessment was limited to height and weight. No adverse events were reported.

Implementation measures. Engagement was objectively assessed as attendance at in-class sessions (yes/no) and completion of IVR calls (yes/no). Implementation fidelity was measured using a structured checklist customized for session learning objectives and assessed as a proportion completion.

Costs were assessed from a community program implementation perspective. Local unit costs were used to value resources to reflect the medically underserved and economic status of the region [69]. Subtotals were broadly categorized as variable costs (i.e., personnel cost) and fixed program costs. Personnel costs were reported based on 
observed hourly rate for program staff and amount of time for training, program preparation, program implementation, and participant management. Similarly, the observed monthly stipend for PAT members to assist in family retention and engagement efforts were included. Fixed program costs (i.e., materials, IVR maintenance fees, facility costs) were also based on the observed costs in the targeted region. Fees charged by one community organization ( $\$ 51$ per facility use time) that were not otherwise subcontracted on the study were used to estimate facility costs. Basic resources and equipment to run the programs (e.g. physical activity equipment, computer, projector) were not included in cost estimates, as it was assumed the facilities would have these resources.

\section{Analysis}

All data were entered into SPSS statistical analyses software (version 21.0, 2012, International Business Machines Corporation, Pittsburgh, PA). Descriptive statistics were used to summarize demographic characteristics, engagement, fidelity, and costs. Chi-square tests (categorical variables) and ANOVA tests (continuous variables) were used to compare demographics between conditions. Outcome variable scores were computed using previously validated scoring procedures. Height and weight data were calculated into BMI z-scores for children and BMI scores for parents.

Quantitative data was examined for presence of outliers, violations of normality (for those continuous variables) and missing data. No major violation of normality was detected. Missing data was addressed using multiple methods, including last-observation-carried-forward, multiple imputation by chained equations, and Heckman selection model [70-73]. For both child BMI z-score and parent BMI levels, Heckman selection bias tests failed to reject the null that the model errors in selection and outcome stages are not correlated. This indicates that observed attrition does not have statistically significant evidence of nonrandomness. The Heckman selection model is a method that directly models the attrition process and provides a direct way to test the existence of nonrandom selection. As such, we present results from the Heckman selection model due to the random nature of missing data and remarkably similar findings for primary outcomes using the other analytical approaches.

In order to detect BMI z-score reduction differences between the two conditions, estimation models all control for errors of non-independence and heteroskedasticity caused by individual and family heterogeneity and cohort clustering (through bootstrapping methods), and a-priori determined covariates. Data from this trial are in longitudinal form, with baseline, 3-, and 6-month time points for primary BMI related outcomes, and baseline and 6-month time points for secondary outcomes. Heckman selection models control race dummies, treatment group indicator, time period indicators and their interactions. In addition, Heckman selection stage models control caregiver's race, education level and employment status which have shown to influence program engagement and outcomes.

Personnel, other, and total costs were summarized descriptively for each cohort, along with costs for all three cohorts. Since PAT members did not lead delivery of any intervention content, this support component may be viewed as optional for future organizations adopting either FBCO program. Likewise, depending on available resources and partnerships, future organizations may or may not incur facility fees when adopting either program. For these reasons and to enhance potential generalizability of cost information presented in this study, three cost estimates are provided: 1) with PAT and with facility costs, 2) without PAT but with facility costs, and 3) without PAT or facility costs. Finally, estimates were calculated as the cost per child enrolled and cost per children with improved BMI z-score.

\section{Results}

\section{Demographics}

Table 1 details child and parent demographics, overall, and by randomized condition. As previously described (Fig. 1), 70 and 69 families were randomized into iChoose and Family Connections, respectively. Eleven families had two children enrolled (i.e., $\mathrm{n}=139$ children and 128 parents). With the exception of a significantly higher proportion of girls being 
randomized into iChoose, there were no other statistically significant demographic difference between conditions. In

brief, children had a mean BMI z-score of $1.93 \pm 0.48$ and mean BMI percentile of $95.95 \pm 3.84$, with the majority (70\%) of children classified as obese. Children had a mean age of $10.1 \pm 1.7$ and were $58 \%$ female, $45 \%$ black and $48 \%$ white, and $9 \%$ Hispanic ethnicity. Additionally, the majority of children were on Medicaid (62\%). 
Table 1

Baseline demographic characteristic of enrolled children and parents, overall and by randomized condition

\begin{tabular}{|c|c|c|c|c|}
\hline & Overall & iChoose & $\begin{array}{l}\text { Family } \\
\text { Connections }\end{array}$ & $\begin{array}{l}\text { Difference between } \\
\text { conditions } \\
\text { test statistic and p-values }\end{array}$ \\
\hline CHILDREN & $\mathrm{n}=139$ & $n=70$ & $n=69$ & \\
\hline BMI & & & & $t=0.77, p=0.44$ \\
\hline BMI z-score, M (SD) & $1.93(0.48)$ & $1.96(0.47)$ & $1.90(0.48)$ & $t=0.74, p=0.46$ \\
\hline BMI Percentile, M (SD) & $96.0(3.8)$ & $96.2(3.7)$ & $95.7(4.0)$ & $x^{2}=0.06, p=0.81$ \\
\hline Overweight, \% (n) & $30 \%(41)$ & $30 \%(20)$ & $30 \%(21)$ & \\
\hline Obese, \% (n) & $70 \%(98)$ & $71 \%(50)$ & $70 \%(48)$ & \\
\hline Age (Years), M (SD) & $10.1(1.7)$ & $10.1(1.7)$ & $10.0(1.7)$ & $t=0.33, p=0.74$ \\
\hline Gender & & & & $x^{2}=3.84, p=0.05$ \\
\hline Female, \% (n) & $58 \%(80)$ & $66 \%(46)$ & $49 \%(34)$ & \\
\hline Race & & & & $x^{2}=1.05, p=0.59$ \\
\hline Black, \% (n) & $45 \%(62)$ & $44 \%(31)$ & $45 \%(31)$ & \\
\hline White, \% (n) & $48 \%(66)$ & $50 \%(35)$ & $45 \%(31)$ & \\
\hline Other, \% (n) & $8 \%(11)$ & $6 \%(4)$ & $10 \%(7)$ & \\
\hline Ethnicity & & & & $x^{2}=0.10, p=0.75$ \\
\hline Hispanic, \% (n) & $9 \%(13)$ & $9 \%(6)$ & $10 \%(7)$ & \\
\hline Insurance type & & & & $x^{2}=1.20, p=0.75$ \\
\hline Medicaid, \% (n) & $62 \%(86)$ & $59 \%(41)$ & $65 \%(45)$ & \\
\hline Private, \% (n) & $33 \%(46)$ & $36 \%(25)$ & $30 \%(21)$ & \\
\hline None, \% (n) & $2 \%(3)$ & $3 \%(2)$ & $1 \%(1)$ & \\
\hline Other, \% (n) & $2 \%(3)$ & $1 \%(1)$ & $3 \%(2)$ & \\
\hline PARENTS & $n=128$ & $n=66$ & $n=62$ & \\
\hline \multicolumn{4}{|l|}{ BMI } & $\mathrm{t}=-0.15, \mathrm{p}=0.88$ \\
\hline & & & & $x^{2}=5.70, p=0.06$ \\
\hline
\end{tabular}

Notes: $M=$ Mean; SD = Standard Deviation; categories do not always equal $100 \%$ due to rounding and some missing responses

${ }^{a}$ t-tests were used to compare means across conditions and $\chi^{2}$ tests were used to compare proportions across the conditions

${ }^{b}$ Employed includes full-time, part-time and self-employment; Unemployed-not in work force includes Homemaker, student, retired, disabled

CHealth literacy was assessed using the validated Newest Vital Sign: High likelihood limited HL (score 0-1), Possibility limited HL (score 2-3), Adequate HL (score 4-6) 


\begin{tabular}{|c|c|c|c|c|}
\hline & Overall & iChoose & $\begin{array}{l}\text { Family } \\
\text { Connections }\end{array}$ & $\begin{array}{l}\text { Difference between } \\
\text { conditions } \\
\text { test statistic and p-values }{ }^{a}\end{array}$ \\
\hline BMI, M (SD) & $\begin{array}{l}36.98 \\
(8.94)\end{array}$ & $\begin{array}{l}36.86 \\
(9.72)\end{array}$ & $37.10(8.11)$ & \\
\hline Normal, \% (n) & $7 \%(9)$ & $12 \%(8)$ & $2 \%(1)$ & \\
\hline Overweight, \% (n) & $17 \%(21)$ & $14 \%(9)$ & $20 \%(12)$ & \\
\hline Obese, \% (n) & $76 \%(97)$ & $74 \%(49)$ & $79 \%(48)$ & \\
\hline Age (Years), M (SD) & $38.9(8.4)$ & $38.8(7.2)$ & $39.0(9.6)$ & $t=-0.11, p=0.91$ \\
\hline Gender & & & & $x^{2}=0.00, p=0.96$ \\
\hline Female, \% (n) & $97 \%(124)$ & $97 \%(64)$ & $97 \%(60)$ & \\
\hline Race & & & & $x^{2}=0.46, p=0.80$ \\
\hline Black, \% (n) & $49 \%(62)$ & $50 \%(33)$ & $48 \%(29)$ & \\
\hline White, \% (n) & $49 \%(62)$ & $49 \%(32)$ & $49 \%(30)$ & \\
\hline Other, \% (n) & $2 \%(3)$ & $2 \%(1)$ & $2 \%(2)$ & \\
\hline Ethnicity & & & & $x^{2}=0.01, p=0.91$ \\
\hline Hispanic, \% (n) & $5 \%(6)$ & $5 \%(3)$ & $5 \%(3)$ & \\
\hline Marital status & & & & $x^{2}=0.06, p=0.97$ \\
\hline Married, \% (n) & $43 \%(54)$ & $42 \%(27)$ & $44 \%(27)$ & \\
\hline Single parent, \% (n) & $55 \%(69)$ & $56 \%(36)$ & $54 \%(33)$ & \\
\hline Unmarried couple, \% (n) & $2 \%(2)$ & $2 \%(1)$ & $2 \%(1)$ & \\
\hline Employment status ${ }^{b}$ & & & & $x^{2}=1.51, p=0.47$ \\
\hline Employed, \% (n) & $68 \%(86)$ & $72 \%(47)$ & $63 \%(39)$ & \\
\hline Unemployed-out of work, \% (n) & $10 \%(13)$ & $8 \%(5)$ & $13 \%(8)$ & \\
\hline $\begin{array}{l}\text { Unemployed-not in work force, \% } \\
\text { (n) }\end{array}$ & $22 \%(28)$ & $20 \%(13)$ & $24 \%(15)$ & \\
\hline Income status & & & & $x^{2}=5.26, p=0.15$ \\
\hline Less than $\$ 20,000$, $\%$ (n) & $29 \%(33)$ & $21 \%(13)$ & $37 \%(20)$ & \\
\hline \multicolumn{5}{|c|}{$\begin{array}{l}\text { Notes: } M=\text { Mean; SD = Standard Deviation; categories do not always equal } 100 \% \text { due to rounding and some missing } \\
\text { responses }\end{array}$} \\
\hline \multicolumn{5}{|c|}{$\begin{array}{l}\text { at-tests were used to compare means across conditions and } \chi^{2} \text { tests were used to compare proportions across the } \\
\text { conditions }\end{array}$} \\
\hline \multicolumn{5}{|c|}{$\begin{array}{l}\text { bEmployed includes full-time, part-time and self-employment; Unemployed-not in work force includes Homemaker, } \\
\text { student, retired, disabled }\end{array}$} \\
\hline $\begin{array}{l}\text { CHealth literacy was assessed usi } \\
\text { Possibility limited HL (score } 2-3 \text { ) }\end{array}$ & $\begin{array}{l}\text { he validate } \\
\text { equate } \mathrm{HL}\end{array}$ & $\begin{array}{l}\text { ewest Vital } \\
\text { re } 4-6)\end{array}$ & n: High likelih & ted HL (score 0-1), \\
\hline
\end{tabular}




\begin{tabular}{|c|c|c|c|c|}
\hline & Overall & iChoose & $\begin{array}{l}\text { Family } \\
\text { Connections }\end{array}$ & $\begin{array}{l}\text { Difference between } \\
\text { conditions } \\
\text { test statistic and p-values }\end{array}$ \\
\hline$\$ 20,000-\$ 44,999, \%(n)$ & $37 \%(43)$ & $41 \%(25)$ & $33 \%(18)$ & \\
\hline$\$ 45,000-\$ 54,999, \%(n)$ & $10 \%(12)$ & $15 \%(9)$ & $6 \%(3)$ & \\
\hline$\$ 55,000$ or more, \% (n) & $24 \%(27)$ & $23 \%(14)$ & $24 \%(13)$ & \\
\hline \multicolumn{4}{|l|}{ Insurance type } & \multirow[t]{6}{*}{$x^{2}=2.02, p=0.73$} \\
\hline Medicaid, \% (n) & $31 \%(39)$ & $29 \%(19)$ & $32 \%(20)$ & \\
\hline Private, \% (n) & $50 \%(63)$ & $54 \%(35)$ & $45 \%(28)$ & \\
\hline None, \% (n) & $9 \%(12)$ & $9 \%(6)$ & $10 \%(6)$ & \\
\hline Medicare, \% (n) & $6 \%(7)$ & $3 \%(2)$ & $8 \%(5)$ & \\
\hline Other & $5 \%(6)$ & $5 \%(3)$ & $5 \%(3)$ & \\
\hline \multicolumn{4}{|l|}{ Education level } & \multirow[t]{5}{*}{$x^{2}=4.93, p=0.18$} \\
\hline $\begin{array}{l}\text { Less than high school diploma, \% } \\
\text { (n) }\end{array}$ & $10 \%(13)$ & $6 . \%(4)$ & $15 \%(9)$ & \\
\hline High school diploma/GED, \% (n) & $20 \%(25)$ & $25 \%(16)$ & $15 \%(9)$ & \\
\hline Some college, \% (n) & $33 \%(42)$ & $29 \%(19)$ & $38 \%(23)$ & \\
\hline College graduate, \% (n) & $37 \%(46)$ & $40 \%(26)$ & $33 \%(20)$ & \\
\hline \multicolumn{4}{|l|}{ Health Literacy $(\mathrm{HL})^{\mathrm{c}}$} & \multirow{5}{*}{$\begin{array}{l}t=-0.01, p=0.99 \\
x^{2}=0.73, p=0.69\end{array}$} \\
\hline NVS (0-6 scale), M (SD) & $4.1(1.8)$ & $4.1(1.8)$ & $4.1(1.8)$ & \\
\hline High likelihood limited HL, \% (n) & $11 \%(13)$ & $10 \%(6)$ & $12 \%(7)$ & \\
\hline Possibility limited HL, \% (n) & $18 \%(22)$ & $21 \%(13)$ & $15 \%(9)$ & \\
\hline Adequate HL, \% (n) & $72 \%(88)$ & $70 \%(44)$ & $73 \%(44)$ & \\
\hline \multicolumn{5}{|c|}{$\begin{array}{l}\text { Notes: } M=\text { Mean; SD = Standard Deviation; categories do not always equal } 100 \% \text { due to rounding and some missing } \\
\text { responses }\end{array}$} \\
\hline \multicolumn{5}{|c|}{$\begin{array}{l}\text { at-tests were used to compare means across conditions and } \chi^{2} \text { tests were used to compare proportions across the } \\
\text { conditions }\end{array}$} \\
\hline \multicolumn{5}{|c|}{$\begin{array}{l}\text { bEmployed includes full-time, part-time and self-employment; Unemployed-not in work force includes Homemaker, } \\
\text { student, retired, disabled }\end{array}$} \\
\hline \multicolumn{5}{|c|}{$\begin{array}{l}\text { 'Health literacy was assessed using the validated Newest Vital Sign: High likelihood limited HL (score 0-1), } \\
\text { Possibility limited HL (score 2-3), Adequate HL (score 4-6) }\end{array}$} \\
\hline
\end{tabular}

Parents had a mean BMI of $36.98 \pm 8.94$ and most were overweight (17\%) or obese $(76 \%)$. On average, parents were 38.9 \pm 8.4 years of age. Nearly all enrolled parents were female (97\%), about half were single parents (55\%), and most were employed (68\%). In terms of income, roughly one-third of parents reported income in each of the following categories: less than $\$ 20,000, \$ 20,000-45,000$, or greater than $\$ 45,000$. The most common reported forms of insurance were private (50\%) and Medicaid (31\%). Finally, $30 \%$ of parents had a high school education or less and $28 \%$ had a high 
likelihood or possibility of limited health literacy. When comparing families that were eligible and enrolled to those that were eligible and declined, families that enrolled had children with significantly greater weight status (95th vs 96th percentile), that were older (10.0 vs 9.4 years), and that were less likely to be white ( $58 \%$ vs $49 \%$; all $p<0.05)$.

Effectiveness

Children BMI z-score 6-month changes from baseline was not statistically significant within iChoose $[0.03(95 \% \mathrm{Cl}=$ $-0.13,0.19)$ ] or Family Connections [BMI z-score $0.00(95 \% \mathrm{Cl}=-0.16,0.16)$ ](Fig. 3a). Similar null findings were found for parents' BMI 6-month changes within iChoose [BMI - $1.04(95 \% \mathrm{Cl}=-4.15,2.08)]$ and Family Connections [BMI $0.04(95 \%$ $\mathrm{Cl}=-2.95,3.02)](F i g .3 b)$. Likewise, relative between condition over time effects was not significant for children BMI zscore or parents BMI level at either 3- or 6-months.

Figure 4 illustrates the proportion of children and parents within each program that had a 6-month decrease, increases, or missing BMI data. Descriptively, a greater proportion of Family Connections children (49\%) and parents (42\%) experienced 6-month decreases in BMI z-scores and BMI, relative to iChoose children (34\%) and parents (32\%). Meanwhile, a higher proportion of iChoose children (37\%) and parents (39\%) had missing BMI z-score data, reflecting higher program attrition, when compared to Family Connections children (16\%) and parents (23\%).

Table 2 describes baseline to 6-month changes in child and parent secondary outcomes. Among parents, an average 6.9 unhealthy days $(95 \% \mathrm{Cl}=0.6,13.2, \mathrm{p}=0.03)$ differences were observed between conditions over time, with improvements among Family Connections parents (e.g. -3.8 unhealthy days and significant at $10 \%$ level) and non-statistically significant increase of 3.1 unhealthy days for iChoose parents. Additionally, 6-month changes at $10 \%$ significance level, on average, indicate: iChoose children decreased sugary beverage consumption; Family Connection children improve total QOL, psychosocial QOL, and fruit and vegetable intake; iChoose parents increased fruit and vegetable intake and increased moderate and vigorous physical activity levels. 
Table 2

Changes in secondary outcomes among children and parents, by randomized treatment condition

\begin{tabular}{|c|c|c|c|c|c|c|c|c|c|c|}
\hline \multirow[t]{4}{*}{ Variable } & \multicolumn{4}{|c|}{ iChoose } & \multicolumn{4}{|c|}{ Family Connections } & \multirow{2}{*}{\multicolumn{2}{|c|}{$\begin{array}{l}\text { Relative effects } \\
\text { between } \\
\text { conditions }^{b}\end{array}$}} \\
\hline & \multirow{3}{*}{$\begin{array}{l}\begin{array}{l}\text { Base- } \\
\text { line }^{a}\end{array} \\
\text { Mean } \\
\text { (SD) }\end{array}$} & \multirow{2}{*}{$\begin{array}{l}\text { 6- } \\
\text { montha }\end{array}$} & \multirow{2}{*}{\multicolumn{2}{|c|}{$\begin{array}{l}\text { Adjusted } \\
\text { change } \\
\text { baseline to 6- } \\
\text { month }^{b}\end{array}$}} & \multirow{2}{*}{$\begin{array}{l}\text { Base- } \\
\text { line }\end{array}$} & \multirow{2}{*}{$\begin{array}{l}\text { 6- } \\
\text { montha }\end{array}$} & \multirow{2}{*}{\multicolumn{2}{|c|}{$\begin{array}{l}\begin{array}{l}\text { Adjusted } \\
\text { change }\end{array} \\
\text { baseline to 6- } \\
\text { month }^{\mathrm{b}}\end{array}$}} & & \\
\hline & & & & & & & & & & \\
\hline & & $\begin{array}{l}\text { Mean } \\
\text { (SD) }\end{array}$ & $\begin{array}{l}\text { Coeff } \\
(95 \% \\
\text { Cl) }\end{array}$ & $\begin{array}{l}\text { p- } \\
\text { value }\end{array}$ & $\begin{array}{l}\text { Mean } \\
\text { (SD) }\end{array}$ & $\begin{array}{l}\text { Mean } \\
\text { (SD) }\end{array}$ & $\begin{array}{l}\text { Coeff } \\
\left(\begin{array}{l}95 \% \\
\text { Cl) }\end{array}\right.\end{array}$ & $\begin{array}{l}\text { p- } \\
\text { value }\end{array}$ & $\begin{array}{l}\text { Coeff } \\
(95 \% \\
\text { Cl) }\end{array}$ & $\begin{array}{l}\mathrm{p} \text { - } \\
\text { value }\end{array}$ \\
\hline \multicolumn{11}{|l|}{ CHILDREN } \\
\hline \multirow{2}{*}{$\begin{array}{l}\text { Systolic blood } \\
\text { pressure, } \\
\mathrm{mm} \mathrm{Hg}\end{array}$} & \multirow{2}{*}{$\begin{array}{l}107.8 \\
(11.3)\end{array}$} & \multirow{2}{*}{$\begin{array}{l}108.3 \\
(10.9)\end{array}$} & 0.7 & \multirow[t]{2}{*}{0.79} & 104.8 & \multirow{2}{*}{$\begin{array}{l}107.3 \\
(11.1)\end{array}$} & 2.213 & \multirow[t]{2}{*}{0.31} & -1.6 & \multirow[t]{2}{*}{0.63} \\
\hline & & & $\begin{array}{l}(-4.1 \\
5.4)\end{array}$ & & $(11.2)$ & & $\begin{array}{l}(-2.0 \\
6.5)\end{array}$ & & $\begin{array}{l}(-7.9 \\
4.8)\end{array}$ & \\
\hline \multirow{3}{*}{$\begin{array}{l}\text { Diastolic } \\
\text { blood } \\
\text { pressure, } \\
\mathrm{mm} \mathrm{Hg}\end{array}$} & \multirow{3}{*}{$\begin{array}{l}66.3 \\
(9.7)\end{array}$} & 67.6 & 1.4 & \multirow[t]{3}{*}{0.48} & \multirow{3}{*}{$\begin{array}{l}64.5 \\
(11.1)\end{array}$} & \multirow{3}{*}{$\begin{array}{l}65.2 \\
(9.6)\end{array}$} & 0.6 & \multirow[t]{3}{*}{0.76} & 0.8 & \multirow[t]{3}{*}{0.73} \\
\hline & & $(8.1)$ & $\begin{array}{l}(-2.4 \\
5.1)\end{array}$ & & & & $\begin{array}{l}(-3.2 \\
4.3)\end{array}$ & & $\begin{array}{l}(-3.6 \\
5.2)\end{array}$ & \\
\hline & & & & & & & & & & \\
\hline \multirow{2}{*}{$\begin{array}{l}\text { Quality of Life: } \\
\text { Total }^{C}\end{array}$} & \multirow{2}{*}{$\begin{array}{l}69.2 \\
(14.1)\end{array}$} & \multirow{2}{*}{$\begin{array}{l}68.4 \\
(14.8)\end{array}$} & -0.7 & \multirow[t]{2}{*}{0.83} & \multirow{2}{*}{$\begin{array}{l}66.9 \\
(16.4)\end{array}$} & \multirow{2}{*}{$\begin{array}{l}71.9 \\
(15.0)\end{array}$} & 5.0 & \multirow[t]{2}{*}{0.07} & -5.7 & \multirow[t]{2}{*}{0.21} \\
\hline & & & $\begin{array}{l}(-7.3 \\
5.9)\end{array}$ & & & & $\begin{array}{l}(-0.5 \\
10.4)\end{array}$ & & $\begin{array}{l}(-14.5, \\
3.2)\end{array}$ & \\
\hline \multirow{2}{*}{$\begin{array}{l}\text { Quality of Life: } \\
\text { Physicalc }\end{array}$} & $\begin{array}{l}73.2 \\
(154)\end{array}$ & 74.8 & 1.7 & 0.62 & 70.8 & 75.1 & 4.1 & 0.13 & -2.4 & 0.60 \\
\hline & & & $\begin{array}{l}(-5.0 \\
8.4)\end{array}$ & & & & $\begin{array}{l}(-1.2 \\
9.4)\end{array}$ & & $\begin{array}{l}(-11.5, \\
6.7)\end{array}$ & \\
\hline Quality of Life: & 67.1 & 65.0 & -2.0 & 0.58 & 64.8 & 70.0 & 5.1 & 0.09 & -7.1 & 0.13 \\
\hline Psychosocial $^{\mathrm{C}}$ & & & $\begin{array}{l}(-9.1 \\
5.1)\end{array}$ & & & & $\begin{array}{l}(-0.8 \\
11.0)\end{array}$ & & $\begin{array}{l}(-16.4, \\
2.1)\end{array}$ & \\
\hline Nutrition: & $\begin{array}{l}3.7 \\
(32)\end{array}$ & 3.3 & -0.3 & 0.67 & 2.9 & 3.7 & 0.9 & 0.07 & -1.1 & 0.18 \\
\hline $\begin{array}{l}\text { vegetables, } \\
\text { serving/day }\end{array}$ & & (3.0) & $\begin{array}{l}(-1.5 \\
1.0)\end{array}$ & & $(2.5)$ & $(4.0)$ & $\begin{array}{l}(-0.1 \\
1.8)\end{array}$ & & $\begin{array}{l}(-2.8 \\
0.5)\end{array}$ & \\
\hline $\begin{array}{l}\text { Nutrition: } \\
\text { Water ounces/ }\end{array}$ & 16.4 & 21.7 & 5.5 & 0.21 & 20.9 & 22.0 & 1.383 & 0.72 & 4.1 & 0.48 \\
\hline $\begin{array}{l}\text { Water, ounces/ } \\
\text { day }\end{array}$ & & & $\begin{array}{l}(-3.0 \\
14.1)\end{array}$ & & $(18.2)$ & & $\begin{array}{l}(-6.060 \\
8.825)\end{array}$ & & $\begin{array}{l}(-7.3 \\
15.6)\end{array}$ & \\
\hline
\end{tabular}

\footnotetext{
a Means (Standard Deviations) are not adjusted for covariates.

${ }^{b}$ Models controlled for race and the selection stage controlled for parental race, parental education level and parental employment status. The $95 \%$ confidence intervals are adjusted to be cohort robust through boostrapping procedure. The estimation is intention-to-treat Heckman selection models.

${ }^{c}$ Unit is percent (0-100), higher scores indicate better health related quality of life.

$\mathrm{d}$ Unit is number of unhealthy days in last 30 days, high scores indicate worse quality of life.
} 


\begin{tabular}{|c|c|c|c|c|c|c|c|c|c|c|}
\hline \multirow[t]{2}{*}{ Variable } & \multicolumn{4}{|l|}{ iChoose } & \multicolumn{4}{|c|}{ Family Connections } & \multirow{2}{*}{\multicolumn{2}{|c|}{$\begin{array}{l}\text { Relative effects } \\
\text { between } \\
\text { conditions }^{\text {b }}\end{array}$}} \\
\hline & $\begin{array}{l}\text { Base- } \\
\text { line }^{a}\end{array}$ & $\begin{array}{l}\text { 6- } \\
\text { month }\end{array}$ & \multicolumn{2}{|c|}{$\begin{array}{l}\text { Adjusted } \\
\text { change } \\
\text { baseline to 6- } \\
\text { monthb }^{\text {b }}\end{array}$} & $\begin{array}{l}\text { Base- } \\
\text { line }^{a}\end{array}$ & $\begin{array}{l}\text { 6- } \\
\text { montha }\end{array}$ & \multicolumn{2}{|c|}{$\begin{array}{l}\text { Adjusted } \\
\text { change } \\
\text { baseline to 6- } \\
\text { monthb }^{\text {b }}\end{array}$} & & \\
\hline \multirow{2}{*}{$\begin{array}{l}\text { Nutrition: } \\
\text { Sugary } \\
\text { beverages, } \\
\text { ounces/ day }\end{array}$} & \multirow{2}{*}{$\begin{array}{l}24.1 \\
(34.5)\end{array}$} & \multirow{2}{*}{$\begin{array}{l}15.8 \\
(18.6)\end{array}$} & -8.0 & \multirow[t]{2}{*}{0.06} & \multirow{2}{*}{$\begin{array}{l}17.7 \\
(18.0)\end{array}$} & \multirow{2}{*}{$\begin{array}{l}13.3 \\
(22.6)\end{array}$} & -4.7 & \multirow[t]{2}{*}{0.25} & -3.3 & \multirow[t]{2}{*}{0.59} \\
\hline & & & $\begin{array}{l}(-16.4 \\
0.3)\end{array}$ & & & & $\begin{array}{l}(-12.8 \\
3.3)\end{array}$ & & $\begin{array}{l}(-15.3 \\
8.6)\end{array}$ & \\
\hline \multirow{2}{*}{$\begin{array}{l}\text { Physical } \\
\text { activity: MVPA, } \\
\text { min/week }\end{array}$} & 192.0 & \multirow{2}{*}{$\begin{array}{l}168.6 \\
(185.9)\end{array}$} & -30.4 & \multirow[t]{2}{*}{0.43} & 174.4 & 195.5 & 16.3 & \multirow[t]{2}{*}{0.67} & -46.6 & \multirow[t]{2}{*}{0.41} \\
\hline & $(242.0)$ & & $\begin{array}{l}(-106.2 \\
45.5)\end{array}$ & & $(232.4)$ & $(215.3)$ & $\begin{array}{l}(-58.0 \\
90.5)\end{array}$ & & $\begin{array}{l}(-157.6 \\
64.3)\end{array}$ & \\
\hline \multicolumn{11}{|l|}{ PARENTS } \\
\hline \multirow{2}{*}{$\begin{array}{l}\text { Waist } \\
\text { Circumference, } \\
\mathrm{cm}\end{array}$} & $\begin{array}{l}110.7 \\
(20 ?)\end{array}$ & $\begin{array}{l}109.9 \\
(197)\end{array}$ & -0.6 & 0.89 & $\begin{array}{l}111.8 \\
(161)\end{array}$ & $\begin{array}{l}113.5 \\
(168)\end{array}$ & 1.6 & 0.66 & -2.1 & 0.69 \\
\hline & & & $\begin{array}{l}(-8.6 \\
7.5)\end{array}$ & & & & $\begin{array}{l}(-5.4 \\
8.5)\end{array}$ & & $\begin{array}{l}(-12.4 \\
8.1)\end{array}$ & \\
\hline $\begin{array}{l}\text { Systolic blood } \\
\text { pressure }\end{array}$ & $\begin{array}{l}122.3 \\
(18.3)\end{array}$ & $\begin{array}{l}124.7 \\
(21.2)\end{array}$ & & 0.42 & $\begin{array}{l}124.0 \\
(210)\end{array}$ & $\begin{array}{l}121.7 \\
(174)\end{array}$ & -2.1 & 0.57 & 5.1 & 0.37 \\
\hline $\mathrm{mm} \mathrm{Hg}$ & & & $\begin{array}{l}(-4.2 \\
10.2)\end{array}$ & & & & $\begin{array}{l}(-9.2 \\
5.0)\end{array}$ & & $\begin{array}{l}(-6.1 \\
16.3)\end{array}$ & \\
\hline Diastolic & $\begin{array}{l}79.9 \\
\left(11^{7}\right)\end{array}$ & 81.7 & 2.2 & 0.34 & 80.0 & 79.4 & -0.3 & 0.90 & 2.5 & 0.43 \\
\hline $\begin{array}{l}\text { pressure, } \\
\mathrm{mm} \mathrm{Hg}\end{array}$ & & (13.9) & $\begin{array}{l}(-2.3 \\
6.8)\end{array}$ & & & & $\begin{array}{l}(-4.2 \\
3.7)\end{array}$ & & $\begin{array}{l}(-3.7 \\
8.6)\end{array}$ & \\
\hline $\begin{array}{l}\text { Quality of Life: } \\
\# \text { unhealthy }\end{array}$ & 12.6 & $\begin{array}{l}15.7 \\
(113)\end{array}$ & 3.1 & 0.18 & 14.4 & 11.0 & -3.8 & 0.09 & 6.9 & 0.03 \\
\hline $\begin{array}{l}\text { days/month in } \\
\text { past } 30 \text { days }\end{array}$ & & & $\begin{array}{l}(-1.4 \\
7.5)\end{array}$ & & & $(9.8)$ & $\begin{array}{l}(-8.2 \\
0.5)\end{array}$ & & $\begin{array}{l}(0.6 \\
13.2)\end{array}$ & \\
\hline $\begin{array}{l}\text { Nutrition: } \\
\text { Fruits \& }\end{array}$ & 2.8 & 3.6 & 0.8 & 0.07 & 3.0 & 3.0 & -0.0 & 0.98 & 0.9 & 0.17 \\
\hline $\begin{array}{l}\text { vegetables, } \\
\text { servings/day }\end{array}$ & $(3.0)$ & $(2.5)$ & $\begin{array}{l}(-0.1 \\
1.8)\end{array}$ & & $(2.4)$ & $(2.1)$ & $\begin{array}{l}(-0.8 \\
0.8)\end{array}$ & & $\begin{array}{l}(-0.4 \\
2.1)\end{array}$ & \\
\hline Nutrition: & 27.8 & 18.8 & -8.8 & 0.16 & 29.1 & 18.6 & -10.5 & 0.15 & 1.8 & 0.86 \\
\hline $\begin{array}{l}\text { beverages, } \\
\text { ounces/day }\end{array}$ & $(34.1)$ & $(27.7)$ & $\begin{array}{l}(-21.1 \\
3.5)\end{array}$ & & $(36.2)$ & $(26.4)$ & $\begin{array}{l}(-24.9 \\
3.8)\end{array}$ & & $\begin{array}{l}(-17.5 \\
21.1)\end{array}$ & \\
\hline $\begin{array}{l}\text { Nutrition: } \\
\text { Water }\end{array}$ & $\begin{array}{l}31.5 \\
(230)\end{array}$ & & & 0.97 & $\begin{array}{l}29.3 \\
(20.3)\end{array}$ & $\begin{array}{l}33.6 \\
(199)\end{array}$ & 4.4 & 0.26 & -4.5 & 0.38 \\
\hline ounces/day & & $(21.8)$ & $\begin{array}{l}(-7.9 \\
7.6)\end{array}$ & & & & $\begin{array}{l}(-3.3 \\
12.1)\end{array}$ & & $\begin{array}{l}(-14.7 \\
5.6)\end{array}$ & \\
\hline a Means (Stand & Deviat & s) are nc & djusted & r cova & tes. & & & & & \\
\hline $\begin{array}{l}\text { b Models control } \\
\text { parental employ } \\
\text { procedure. The }\end{array}$ & $\begin{array}{l}\text { ed for rac } \\
\text { nent statu } \\
\text { stimation }\end{array}$ & $\begin{array}{l}\text { and the s } \\
\text { The } 95 \% \\
\text { intention }\end{array}$ & $\begin{array}{l}\text { lection st } \\
\text { confidenc } \\
\text { o-treat He }\end{array}$ & $\begin{array}{l}\text { ge cont } \\
\text { interva } \\
\text { kman s }\end{array}$ & $\begin{array}{l}\text { lled for pa } \\
\text { are adjus } \\
\text { lection } \mathrm{m}\end{array}$ & $\begin{array}{l}\text { ental race } \\
\text { ed to be c } \\
\text { dels. }\end{array}$ & $\begin{array}{l}\text { parental } \\
\text { hort robu }\end{array}$ & $\begin{array}{l}\text { ucatio } \\
\text { throus }\end{array}$ & $\begin{array}{l}\text { evel and } \\
\text { boostrap }\end{array}$ & ing \\
\hline${ }^{\mathrm{C}}$ Unit is percent & $-100)$, hi & er scores & dicate $b$ & ter hea & h related & ality of li & & & & \\
\hline
\end{tabular}




\begin{tabular}{|c|c|c|c|c|c|c|c|c|}
\hline \multirow[t]{3}{*}{ Variable } & \multicolumn{3}{|l|}{ iChoose } & \multicolumn{3}{|c|}{ Family Connections } & \multirow{3}{*}{\multicolumn{2}{|c|}{$\begin{array}{l}\text { Relative effects } \\
\text { between } \\
\text { conditions }^{b}\end{array}$}} \\
\hline & \multirow{2}{*}{$\begin{array}{l}\text { Base- } \\
\text { line }^{a}\end{array}$} & \multirow{2}{*}{ montha } & \multirow{2}{*}{$\begin{array}{l}\begin{array}{l}\text { Adjusted } \\
\text { change }\end{array} \\
\text { baseline to 6- } \\
\text { month }^{b}\end{array}$} & \multirow{2}{*}{$\begin{array}{l}\text { Base- } \\
\text { line }^{a}\end{array}$} & \multirow{2}{*}{ montha } & \multirow{2}{*}{$\begin{array}{l}\text { Adjusted } \\
\text { change } \\
\text { baseline to 6- } \\
\text { month }^{b}\end{array}$} & & \\
\hline & & & & & & & & \\
\hline \multirow{2}{*}{$\begin{array}{l}\text { Physical } \\
\text { activity: MVPA, } \\
\text { min/week }\end{array}$} & \multirow{2}{*}{$\begin{array}{l}139.2 \\
(182.4)\end{array}$} & 218.2 & 80.1 & \multirow{2}{*}{$\begin{array}{l}166.8 \\
(212.2)\end{array}$} & 172.2 & 0.88 & 74.4 & \multirow[t]{2}{*}{0.24} \\
\hline & & $(241.3)$ & $\begin{array}{l}(-13.1 \\
173.3)\end{array}$ & & $(182.0)$ & $\begin{array}{l}(-70.8 \\
82.2)\end{array}$ & $\begin{array}{l}(-50.3 \\
199.1)\end{array}$ & \\
\hline \multicolumn{9}{|c|}{ a Means (Standard Deviations) are not adjusted for covariates. } \\
\hline \multicolumn{9}{|c|}{$\begin{array}{l}\text { b Models controlled for race and the selection stage controlled for parental race, parental education level and } \\
\text { parental employment status. The } 95 \% \text { confidence intervals are adjusted to be cohort robust through boostrapping } \\
\text { procedure. The estimation is intention-to-treat Heckman selection models. }\end{array}$} \\
\hline \multicolumn{9}{|c|}{${ }^{c}$ Unit is percent (0-100), higher scores indicate better health related quality of life. } \\
\hline
\end{tabular}

Implementation fidelity

iChoose class components were ranked highly by both independent observers (nutrition sessions $=99 \pm 3 \%$; parent behavioral sessions = $96 \pm 10 \%$; child behavioral sessions $=99 \pm 2 \%$; and maintenance skill building sessions $=98 \pm 7 \%$ ) and self-report by program facilitators (nutrition sessions $=99 \pm 3 \%$; parent behavioral sessions $=99 \pm 2 \%$, child behavioral sessions $=100 \%$; and maintenance skill building sessions $=78 \pm 8 \%$ ). Self-reported fidelity for iChoose physical activity classes indicated $87 \%$ achievement of moderate to vigorous activity goals. Independent observers rated implementation fidelity high for Family Connections classes (99 $\pm 1 \%$ ). Routine weekly monitoring of the IVR software platform indicated $100 \%$ of IVR calls for both programs were sent as intended.

Engagement

iChoose class engagement level averaged $3.6 \pm 3.9$ of 12 family classes (30\%), $4.3 \pm 4.7$ of 12 IVR calls (36\%), and $9.1 \pm$ 12.4 of 36 recommended physical activity classes (25\%). For the 2 parent classes and 10 IVR calls in Family Connections, class attendance averaged $1.0 \pm 0.9$ (52\%) and IVR call completion averaged $6.2 \pm 4.4(61 \%)$. Implementation costs

Figures 5 and 6 provide details on program implementation costs. When considering costs that include both PAT and facility fees, program differences are shown for total costs (iChoose $=\$ 68,289 ;$ Family Connections $=\$ 32,533$; Fig. 5), cost per child enrolled (iChoose $=\$ 976$; Family Connections $=\$ 471$; Fig. 6), and cost per children with improved BMI zscore (iChoose = \$2,845; Family Connections = \$957; Fig. 6). Removal of the PAT reduces costs by about $22 \%$ for both programs, while removal of the PAT and facility costs reduces total cost by about $53 \%$ for iChoose and $27 \%$ for Family Connections.

\section{Discussion}

Our FBCO treatment study was designed and executed within a local community context, taking into consideration the available resources and infrastructure of local health care and community organizations in a health disparate region. Our study setting and sample is distinct from most other published FBCO treatment trials that were typically conducted in urban academic medical centers or large primary care settings and that include samples with higher SES, less ethnic/minority representation, and a higher proportion of married household [74-77]. Our concurrent appraisal of reach, effectiveness, and implementation outcomes of two FBCO interventions within a medically underserved region 
addresses notable gaps in the scientific literature and helps guide stakeholder decisions pertaining to organizational maintenance of an intervention.

Contrary to our hypotheses, the high intensity iChoose program did not yield significantly greater improvements in child BMI z-scores as compared to the low intensity Family Connections program. Despite exceptionally high implementation fidelity ratings for both iChoose and Family Connections, neither program yielded significant improvements in child BMI z-scores or parent BMI levels. In previously published trials of Bright Bodies [18-20] (from which iChoose was adapted from) and Family Connections [35], BMI z-score reductions were around 0.16 and 0.07 respectively. Our findings highlight the ability to achieve high fidelity when systematically translating FBCO treatment interventions for adoption and implementation by community partners; but also highlight challenges of replicating effects established among urban and relatively affluent communities into a more rural, low SES, and medically underserved community.

Direct evidence on a clinically significant threshold for BMI z-score changes among overweight and obese children is not clearly established. Various reports and expert panels typically suggest BMI z-score reductions at benchmarks of 0.25 , 0.20 , and 0.15 as clinically significant and as associated with improvements in cardiometablic profile $[22,78]$. While other reviews offer differing interpretations of clinically relevant BMI z-score improvements (e.g., 0.12-0.16) [79]. Notably, arresting gain in excess BMI also likely constitutes a clinically important benefit for many children $[76,78,80]$. The clinical significance of achieving weight stabilization may be especially relevant among overweight children (which includes about $1 / 3$ of the children in our study) and among communities that bear unequal burden of childhood obesity, such as the minority and low income families targeted in this trial. Of studies used to inform the USPSTF childhood obesity treatment recommendations, few trials targeted more rural, minority, and low-income communities [22]. Coupled with our null findings in this type of disparate region, it is important to identify similar treatment recommendations to tackle childhood obesity in those areas.

Other trials that have examined childhood obesity treatment effects by programs of varying structure and intensity have been mixed, with some observing better effects for high intensity programs [77] and others observing no differences [74-76]. Our study findings on QOL, proportion of participants with BMI improvements, retention, and engagement suggest that a lower intensity program may better fit the needs of our intended audience. Specifically, Family Connections parents improved QOL, relative to iChoose parents. A greater proportion of Family Connections children and parents experienced 6-month BMI improvements compared to iChoose families. Though this finding is only descriptive and should be interpreted with caution. Also, 6-month retention rates were higher for Family Connection (84\%) than for iChoose (63\%) families. Finally, engagement in Family Connections components (52-61\%) was higher than engagement in iChoose components (25-36\%).

Challenges in engaging families in childhood obesity treatment interventions are well documented in the literature [23$29,81]$. Low engagement reduces participant exposure to intended program content. Despite use of a PAT to provide a social network and serve as a safety net to promote family engagement in our CER trial, our engagement remained substantially lower than desired. It is difficult to make strong inferences about how family engagement may have been impacted without a PAT, yet we postulate that engagement may have suffered even more. Compared to our previous single-group 3-month pilot trial of iChoose $(n=101$ families, retention $=72 \%, 43 \%$ family class attendance, $62 \%$ call completion, 33\% physical activity class attendance) in this region [30], we were not any more successful with the addition of a PAT and other deliberate engagement and retention strategies for this trial-suggesting that program features may be stronger predictors of engagement than the social support strategies targeting engagement.

Challenges with program engagement and retention could be linked to our approach that focused on optimizing reach to increase the likelihood that those who could benefit most from a FBCO intervention. In our trial, there were few exclusion criteria, active outreach to a large population of families (i.e., not just families motivated enough to respond to passive 
recruitment approaches), and strategies to make it as easy as possible for families to enroll. This can be contrasted with published FBCO trials that use run in periods, have strict standards for enrolling families (e.g., must make a full commitment to engage in all intervention components) and for dismissing families who are not engaging (e.g., missed more than two classes) [82]. These procedures may improve receipt of program content among retained families, and subsequently bolster program effectiveness. However, this approach may not be appropriate for low income families nor be reflective of the service model and mission of typical clinical and community partner organizations who are ultimately intended to adopt and sustain a FBCO treatment intervention.

The economic impact of childhood obesity is complex and the heterogeneity of treatment studies and healthcare models makes it difficult to compare and contrast costs across interventions [83]. Regardless, costs of childhood obesity treatment options helps guide decision making among policy makers, program planners, and community organizations who may choose to adopt a FBCO program. Other cost effectiveness analyses of childhood obesity treatment interventions indicate mixed findings [84-87]. One analysis of 10 RCTs demonstrated that it may take six or seven decades to realize cost savings and health benefits of these interventions [85]. While a full cost effectiveness analysis was beyond the scope of this study, capturing organizational-level program implementation costs and costs per participant with improved BMI outcomes from a budgetary impact perspective is an important metric for stakeholders and is rarely reported in other FBCO trials [88]. This metric could be used to compare across studies in the future. We presented actual costs of program implementation in the Dan River Region, which allow other communities to adjust and project potential costs in different scenarios. For this trial, overall program implementation costs for the higher intensity iChoose program was approximately 2-3 times higher than for the lower intensity Family Connections program. This cost data, along with outcome and engagement data, has been shared with our CAB and will be used to inform future FBCO intervention adaptation and adoption.

Our findings inform several potential future directions for this line of FBCO research. First, development of self-regulation skills through more intensive self-monitoring strategies should be revisited both iChoose and Family Connections. In weight-related trials, evidence-based self-monitoring strategies often target diet, exercise and/or self-weighing [89]. Both our FBCO treatment interventions included several self-monitoring strategies (i.e., IVR component to assist with diet and exercise goal setting and feedback, structured self-monitoring activities within classes and take-home activities for families, bi-weekly weigh-ins for iChoose families). Nonetheless, limited improvements in self-reported behavioral outcomes may suggest insufficient emphasis on these strategies. Second, future efforts are needed to explore additional asynchronous, remote and/or technology-based programmatic options. Relative to in-person components, our study showed higher engagement rates for the asynchronous, but proactive IVR components. For lower resourced families, there is an important balance of building face-to-face relationships and support systems versus meeting the needs of these busy families, including many single headed households. Third, engaging clinical providers at the point of care for families is also a promising approach in the FBCO research literature and should be strongly considered in future efforts $[75,90]$.

When first embarking on this FBCO research over five years ago, our CAB partners helped guide key decisions around self-monitoring strategies (i.e., strong dislike about calorie counting, concerns pertaining to overt focus on weight and regularly weighing children as related to self-esteem and bullying) and program structure (i.e., perceived need for face-toface contact with families). With local data now available on these two FBCO programs, along with the recent surge of internet connectively and ubiquitous utilization of mobile technology, future efforts should consider more robust remote strategies to deliver childhood obesity treatment intervention content, provide support, and assist with goal setting and self-monitoring. These efforts should be guided by emerging evidence from digital and telehealth approaches in other childhood obesity treatment trials [76, 91-93]. Likewise, digital approaches and other novel strategies could also help engage and support other family members and friends who also influence the child's home environment. Finally, it is well documented that numerous genetic, behavioral, and environment factors contribute to increased risk and childhood

Page $16 / 29$ 
obesity disparities [94]. In alignment with socioecological models of childhood obesity, a comprehensive family-centered behavioral intervention may be a necessary, but insufficient component to reverse childhood obesity in a region with a myriad of health inequities. As such, the CAB partnership and structure should be expanded to allow for a more comprehensive community approach focused on addressing childhood obesity inequities [95].

Though beyond the scope of this current paper, a more thorough examination of our CER trial data are also warranted. First, the impact of engagement on outcomes deserves further investigation, as preliminary descriptive analysis reveals emergent patterns of low versus high engaged families across both iChoose and Family Connections programs. Second, individual-level program maintenance could further impact decisions pertaining to superiority of one program over the other (though completion of 12-month maintenance remains uncertain due to the COVID-19 public health). Finally, systematic analysis of mixed-methods interviews collected at data assessment appointments is in progress and will help further elucidate challenges and future opportunities to support family engagement and program effects.

Several study limitations should be noted. First, while our findings contribute to the sparse literature on childhood obesity intervention effect on health disparate regions, it may have limited generalizability beyond the study region. Second, the sample size for self-reported secondary outcomes among children is lower than anticipated and originally powered, due to lowering the inclusion age from 8-12 to 5-12 in the third cohort to meet accrual needs. These limitations should be considered within strengths of the RCT trial design, objective assessment of primary outcomes, appropriate statistical analysis to account for missing data, and robust application of the RE-AIM planning and evaluation framework. Likewise, the systems-based and CBPR approach, whereby research occurred within the existing community infrastructure is a study strength.

\section{Conclusions}

In sum, both FBCO treatment interventions were delivered with similarly high fidelity; yet neither yielded significant improvements in child BMI z-scores or parent BMI scores. Descriptively, relative to the high-intensity iChoose program, the low intensity Family Connections program yielded a higher proportion of families with improved weight outcomes, had higher retention and engagement rates, and was delivered at a lower cost per participant enrolled and lower cost per participant with improved weight outcome. Future research efforts need to address lack of program effects and further explore strategies to leverage higher engagement in lower intensity programs for populations in medically underserved areas. Despite the robust CBPR approach and reliance on established evidence-based interventions, this study exemplifies the challenges of replicating childhood obesity treatment effects established in more affluent and urban settings to medically underserved, rural regions.

\section{Abbreviations}

FBCO

family-based childhood obesity

CER

comparative effectiveness research

RCT

randomized controlled trial

CBPR

community-based participatory research

RE-AIM

reach, effectiveness, adoption, implementation, maintenance

IVR

Page 17/29 
interactive voice response

$\mathrm{CAB}$

community advisory board

PAT

parent advisory team

\section{Declarations}

\section{Ethics approval and consent to participate.}

The University of Virginia's Institutional Review Board approved this research. Parents provided written informed consent and children provided assent at enrollment.

\section{Consent for publication.}

Not applicable.

\section{Availability of data and materials.}

The datasets generated during the current study available from the corresponding author on reasonable request.

\section{Competing interests.}

The authors declare that they have no competing interests.

\section{Funding.}

Research reported in this publication was funded through a Patient-Centered Outcomes Research Institute (PCORI) Award (AD-1602-34489). PCORI was not involved is the design of this study or writing of this manuscript. The statements in this article are solely the responsibility of the authors and do not necessarily represent the views of the Patient-Centered Outcomes Research Institute (PCORI), its Board of Governors or Methodology Committee.

\section{Authors' contributions.}

PE and JZ proposed study hypotheses and secured funding. PE, DB, MY and BP developed curriculum materials. PE, DB, MY, BP, JW and DM were responsible for program implementation and fidelity monitoring. JZ, DB, MY, BP, JW, and DM managed family recruitment and data collection. JZ, WY, JH, DB, MY, BP and PE were responsible for guiding and reviewing interpretation of all process and outcome data reports. WY conducted final statistical analyses. JZ drafted the initial manuscript. All authors read and approved the final manuscript and are personally accountable for the accuracy of this manuscript.

\section{Acknowledgements.}

We would like to recognize CAB members who have been instrumental in the execution of this study: Jason Bookheimer, Beth Thomas, Stephanie Phelps, Deana Jones, Paige Shelton, Kenya Elliott, and Marsha Mendenhall. We would also like to thank all the PAT members for their commitment and enthusiasm for this study. We especially acknowledge the 
unwavering support from our PAT members Wanda Breedlove, Pamela Carter-Taylor, Julie Matejko, Misty Roveta, and Tia Yancy.

\section{References}

1. Ogden CL, Carroll MD, Lawman HG, Fryar CD, Kruszon-Moran D, Kit BK, et al. Trends in obesity prevalence among children and adolescents in the United States, 1988-1994 Through 2013-2014. JAMA. 2016;315(21):2292-9.

2. Bethell C, Simpson L, Stumbo S, Carle AC, Gombojav N. National, state, and local disparities in childhood obesity. Health Aff. 2010;29(3):347-56.

3. Rossen LM, Schoendorf KC. Measuring health disparities: trends in racial- ethnic and socioeconomic disparities in obesity among 2- to 18-year old youth in the United States, 2001-2010. Ann of Epidemiol. 2012; 22(10):698-704.

4. Johnson JA, Johnson AM. Urban-rural differences in childhood and adolescent obesity in the United States: a systematic review and meta-analysis. Childhood Obes. 2015;11(3):233-41.

5. Ogden CL, Carroll MD, Kit BK, Flegal KM. Prevalence of obesity and trends in body mass index among us children and adolescents, 1999-2010. JAMA. 2012; 307(5):483-90.

6. Ogden CL, Carroll MD, Kit BK, Flegal KM. Prevalence of childhood and adult obesity in the United States, 2011-2012. JAMA. 2014; 311(8):806-14.

7. Wilfley D, Staiano A, Altman M, Lindros J, Lima A, Hassink G, et al. Improving access and systems of care for evidence-based childhood obesity treatment: conference key findings and next steps. Obesity. 2017; 25(1):16-29.

8. Wolfenden L, Wiggers J, d'Espaignet ET, Bell AC. how useful are systematic reviews of child obesity interventions? Obes Rev. 2010; 11(2):159-66.

9. Epstein LH, Wing RR, Wood R, Penner BC, Kress MJ, Koeske R. Effects of family-based behavioral treatment on obese 5-to-8 year-old children. Behav Ther. 1985;16(2): 205-12.

10. Epstein LH, Wing, R, Valoski A, Gooding W. Long-term effects of parent weight on child weight loss. Behav Ther. 1987;18(3):219-26.

11. Reinehr T. Effectiveness of lifestyle intervention in overweight children. Proc Nutr Soc. 2011; 70(4):494-505.

12. Reinehr T, de Sousa G, Andler W. Longitudinal analyses among overweight, insulin resistance, and cardiovascular risk factors in children. Obes Res. 2005;13(10):1824-33.

13. Golan M, Crow S. Targeting parents exclusively in the treatment of childhood obesity: long-term results. Obes Res. 2004;12(2):357-61.

14. Golan M, Weizman A, Apter A, Fainaru M. Parents as the exclusive agents of change in the treatment of childhood obesity. Am J Clin Nutr. 1998;67(6):1130-35.

15. Altman M, Wilfley DE. Evidence update on the treatment of overweight and obesity in children and adolescents. J Clin Child Adolesc Psychol. 2015;44(4):521-37.

16. Epstein LH, Valoski A, Wing RR, McCurley J. Ten-year outcomes of behavioral family-based treatment for childhood obesity. Health Psychol. 1994;13(5):373-83.

17. Golan M, Kaufman V, Shahar DR. Childhood obesity treatment: targeting parents exclusively v. parents and children. Br J Nutr 2006;95(5):1008-15.

18. Savoye M, Berry D, Dziura J, Shaw M, Serrecchia JB, Barbetta G, et al. Anthropometric and psychosocial changes in obese adolescents enrolled in a weight management program. J Am Diet Assoc. 2005;105(3):364-70.

19. Savoye M, Nowicka P, Shaw M, Yu S, Dziura J, Chavent G, et al. Long-term results of an obesity program in an ethnically diverse pediatric population. Pediatrics. 2011;127(3):402-10. 
20. Savoye M, Shaw M, Dziura J, Tamborlane WV, Rose P, Guandalini C, et al. Effects of a weight management program on body composition and metabolic parameters in overweight children: a randomized controlled trial. JAMA. 2007;297(24):2697-704.

21. Peirson L, Fitzpatrick-Lewis D, Morrison K, Warren R, Usman Ali M, Raina P. Treatment of overweight and obesity in children and youth: a systematic review and meta-analysis. CMAJ open. 2015;3(1):e35-46.

22. O'Connor EA, Evans CV, Burda BU, Walsh ES, Eder M, Lozano P. Screening for obesity and intervention for weight management in children and adolescents: evidence report and systematic review for the US Preventive Dervices Task Gorce. JAMA. 2017;317(23):2427-44.

23. Cote MP, Byczkowski T, Kotagal U, Kirk S, Zeller M, Daniels S. Service quality and attrition: an examination of a pediatric obesity program. Int J Qual Health Care. 2004;16(2):165-73.

24. Denzer C, Reithofer E, Wabitsch M, Widhalm K. The outcome of childhood obesity management depends highly upon patient compliance. Eur J Pediatr. 2004;163(2):99-104.

25. Kirk S, Zeller M, Claytor R, Santangelo M, Khoury PR, Daniels SR. The relationship of health outcomes to improvement in BMI in children and adolescents. Obes Res. 2005;13(5):876-82.

26. Skelton JA, Beech BM. Attrition in paediatric weight management: a review of the literature and new directions. Obes Rev. 2011;12(5):e273-81.

27. Zeller M, Kirk S, Claytor R, Khoury P, Grieme J, Santangelo M, et al. Predictors of attrition from a pediatric weight management program. J Pediatr. 2004;144(4):466-70.

28. Barlow SE, Ohlemeyer CL. Parent reasons for nonreturn to a pediatric weight management program. Clin Pediatr (Phila). 2006;45(4):355-60.

29. Klesges LM, Williams NA, Davis KS, Buscemi J, Kitzmann KM. External validity reporting in behavioral treatment of childhood obesity: a systematic review. Am J Prev Med. 2012;42(2):185-92.

30. Hill J, Zoellner J, You W, Brock D, Price B, Alexander R, et al. Participatory development and pilot testing of iChoose: an adaptation of an evidence-based pediatric weight management program for community implementation. BMC Public Health. 2019;19(1):122.

31. Mead E, Brown T, Rees K, Azevedo LB, Whittaker V, Jones D, et al. Diet, physical activity and behavioural interventions for the treatment of overweight or obese children from the age of 6 to 11 years. Cochrane Database of Sys Rev. 2017(6).

32. Zoellner JM, Hill J, You W, Brock D, Frisard M, Alexander R, et al. The influence of parental health literacy status on reach, attendance, retention, and outcomes in a family-based childhood obesity treatment program, Virginia, 20132015. Prev Chronic Dis. 2017;14:e87.

33. Brock DP, Estabrooks PA, Hill JL, Barlow ML, Alexander RC, Price BE, et al. Building and Sustaining community capacity to address childhood obesity: a 3-year mixed-methods case study of a community-academic advisory board. Fam Community Health. 2019;42(1):62-79.

34. Zoellner J, Hill JL, Brock D, Barlow ML, Alexander R, Brito F, et al. One-year mixed-methods case study of a community-academic advisory board addressing childhood obesity. Health Promot Pract. 2017;18(6):833-53.

35. Estabrooks PA, Shoup JA, Gattshall M, Dandamudi P, Shetterly S, Xu S. Automated telephone counseling for parents of overweight children a randomized controlled trial. Am J Prev Med. 2009;36(1):35-42.

36. Best A, et al. NCIC working group on translational research and knowledge integration. The language and logic of research transfer: finding common ground. 2005.

37. Estabrooks PA, Glasgow RE. Translating effective clinic-based physical activity interventions into practice. Am J Prev Med. 2006;31(4):S45-S56. 
38. Israel BA, Eng E, Schulz AJ, Parker EA. Methods in community-based participatory research for health. San Francisco: Jossey-Bass; 2005.

39. Minkler M, Wallerstein N (Eds.). Community Based Participatory Research for Health: From process to outcomes, 2nd edition edition. San Francisco: Jossey Bass; 2008.

40. Wallerstein N, Duran B. Community-based participatory research contributions to intervention research: the intersection of science and practice to improve health equity. Am J Public Health. 2010;Suppl 1(Supp1):S40-46.

41. Estabrooks PA, Fisher EB, Hayman LL. What is needed to reverse the trends in childhood obesity? a call to action. Ann of Behav Med. 2008;36(3):209-16.

42. Klesges LM, Estabrooks PA, Dzewaltowski DA, Bull SS, Glasgow RE. Beginning with the application in mind: designing and planning health behavior change interventions to enhance dissemination. Ann Behav Med. 2005;29(2):66-75.

43. Zoellner JM, You W, Hill JL, Brock DP, Yuhas M, Alexander RC, et al. A comparative effectiveness trial of two familybased childhood obesity treatment programs in a medically underserved region: Rationale, design \& methods. Contemp Clin Trials, 2019;84:105801.

44. Virginia Health Opportunity Index. [https://www.vdh.virginia.gov/omhhe/hoi/dashboards/counties] Accessed May 10, 2018.

45. U.S. Department of Health and Human Services Health Resources and Services Administration: Medically Underserved Areas/Populations. U.S. Department of Health and Human Services, Health Resources and Services Administration. data.hrsa.gov/tools/shortage-area/mua-find. Accessed

46. Haskell WL, Kiernan M. Methodologic issues in measuring physical activity and physical fitness when evaluating the role of dietary supplements for physically activity people. Am J Clin Nutr. 2000;72(2):541S-50.

47. Mettler MM, Stone WJ, Herrick AB, Klein DA. Evaluation of a community-based physical activity campaign via the transtheoretical model. Health Promot Pract. 2000;1(4):351-59.

48. BMI Data of School Children. Yanceyville: Caswell County Public School District; 2011.

49. Brito FA, Zoellner JM, Hill J, You W, Alexander R, Hou X, et al. From bright bodies to iChoose: using a CBPR approach to develop childhood obesity intervention materials for rural Virginia SAGE Open. 2019.

50. Alexander R, Brock DP, Estabrooks P, Hill JL, Whitt-Glover MC, Zoellner J. Capacity development and evaluation of a parent advisory team engaged in obesity research. Health Promot Pract. 2019.

51. Swider SM. Outcome effectiveness of community health workers: an integrative literature review. Public Health Nurs. 2002;19(1):11-20.

52. Fouad MN, Johnson RE, Nagy MC, Person SD, Partridge EE. Adherence and retention in Clinical trials: a communitybased approach. Cancer. 2014;120:1106-12.

53. Katula JA, Vitolins MZ, Rosenberger EL, Blackwell C, Espeland MA, Lawlor MS, et al. Healthy living partnerships to prevent diabetes (HELP PD): design and methods. Contemp Clin Trials. 31(1):71-81.

54. Edmunds JM, Beidas RS, Kendall PC. Dissemination and implementation of evidence-based practices: training and consultation as implementation strategies. Clin Psychol. 2013;20(2):152-65.

55. Wallace LS, Rogers ES, Roskos SE, Holiday DB, Weiss BD. Brief report: screening items to identify patients with limited health literacy skills. J Gen Intern Med. 2006;21(8):874-77.

56. Morris NS, MacLean CD, Chew LD, Littenberg B. The single item literacy screener: evaluation of a brief instrument to identify limited reading ability. BMC Fam Pract. 2006;7(1):21.

57. Jeppesen KM, Coyle JD, Miser WF. Screening questions to predict limited health literacy: a cross-sectional study of patients with diabetes mellitus. Ann Fam Med. 2009;7(1):24-31. 
58. Glasgow RE, Klesges LM, Dzewaltowski DA, Estabrooks PA, Vogt TM. Evaluating the impact of health promotion programs: using the RE-AIM framework to form summary measures for decision making involving complex issues. Health Educ Res. 2006; 21(5):688-94.

59. Perloff D, Grim C, Flack J, Frohlich ED, Hill M, McDonald M, Morgenstern BZ. Human blood pressure determination by sphygmanometry. Circulation. 1993;88(5):2460-70.

60. Waist Circumference Measurement Guidelines - Healthcare Professionals [http://www.myhealthywaist.org/fileadmin/pdf/WCMG-Healthcare_Professional.pdf]. Accessed June 12, 2020.

61. Weiss BD, Mays MZ, Martz W, Castro KM, DeWalt DA, Pignone MP, et al. Quick assessment of literacy in primary care: The newest vital sign. Ann Fam Med. 2005;3(6):514-22.

62. Varni JW, Seid M, Rode CA. The PedsQL ${ }^{\mathrm{T}}$ : measurement model for the pediatric quality of life inventory. Med Care. 1999; 37:126-39.

63. Klesges RC, Eck LH, Mellon MW, Fulliton W, Somes GW, Hanson CL. The accuracy of self-reports of physical activity. Med Sci Sports Exerc. 1990;22(5):690-97.

64. Chronic Diseases: The leading cause of death. United States and Mississippi, 1995 and 2001. The Centers for Disease Control and Prevention. 2007.

65. Hedrick VE, Comber DL, Estabrook PA, Savla J, Davy BM. The beverage intake questionnaire: determining initial validity and reliablity. J Am Diet Assoc. 2010;110(8):1227-32.

66. Hedrick VE, Savla J, Comber DL, Flack KD, Estabrooks PA, Nsiah-Kumi PA, et al. Development of a brief questionnaire to assess habitual beverage intake (BEVQ-15): sugar-sweetened beverages and total beverage energy intake. J Acad Nutr Diet. 2012;112(6):840-49.

67. Hill C, MacDougall C, Riebl S, Savla J, Hedrick VE, Davy BM. Comparative validity and test-retest reliability of the BEVQ-15 in children and adolescents. J Acad Nutr Diet. In Press.

68. Godin G, Shephard RJ. A simple method to assess exercise behavior in the community. Can J Appl Sport Sci. 1985;10(3):141-46.

69. James M, Stokes E. Harnessing information for health economics analysis. CRC Press; 2018.

70. Heckman JJ. Sample selection bias as a specification error. Econometrica. 1979;47(1):153-61.

71. Raghunathan TE, Lepkowksi JM, Van Hoewyk J, Solenbeger P. A multivariate technique for multiply imputing missing values using a sequence of regression models. Surv Methodol. 2001;27(1):85-95.

72. Azur MJ, Stuart EA, Frangakis C, Leaf PJ. Multiple imputation by chained equations: what is it and how does it work? Int J Methods in Psychiatr Res. 2011;20(1):40-49.

73. Galimard JE, Chevret S, Curis E, Resche-Rigon M. Heckman imputation models for binary or continuous MNAR outcomes and MAR predictors. BMC Med Res Methodol. 2018;18(1):90.

74. Bergmann K, Mestre Z, Strong D, Eichen DM, Rhee K, Crow S, et al. Comparison of two models of family-based treatment for childhood obesity: a pilot study. Child Obes. 2019;15(2):116-22.

75. Taveras EM, Marshall R, Kleinman KP, Gillman MW, Hacker K, Horan CM, et al. Comparative effectiveness of childhood obesity interventions in pediatric primary care: a cluster-randomized clinical trial. JAMA Pediatr. 2015;169(6):535-42.

76. Taveras EM, Marshall R, Sharifi M, Avalon E, Fiechtner L, Horan C, et al. Comparative effectiveness of clinicalcommunity childhood obesity interventions: a randomized clinical trial. JAMA Pediatr. 2017;171(8):e171325.

77. Wilfley DE, Saelens BE, Stein RI, Best JR, Kolko RP, Schechtman KB, et al. Dose, content, and mediators of familybased treatment for childhood obesity: a multisite randomized clinical trial. JAMA Pediatr. 2017;171(12):1151-59. 
78. Wiegand S, Keller KM, Lob-Corzilius T, Pott W, Reinehr T, Röbl M, et al. Predicting weight loss and maintenance in overweight/obese pediatric patients. Horm Res Paediatr. 2014;82(6):380-87.

79. Elvsaas IKO, Giske L, Fure B, Juvet LK. Multicomponent lifestyle interventions for treating overweight and obesity in children and adolescents: a systematic review and meta-analyses. J Obes. 2017.

80. Goldschmidt AB, Wilfley DE, Paluch RA, Roemmich JN, Epstein LH. Indicated prevention of adult obesity: how much weight change is necessary for normalization of weight status in children? JAMA Pediatr. 2013;167(1):21-26.

81. Brown CL, Dovico J, Garner-Edwards D, Moses M, Skelton JA. Predictors of engagement in a pediatric weight management clinic after referral. Child Obes. 2020;16(4):238-43.

82. Ruebel ML, Heelan KA, Bartee T, Foster N. Outcomes of a family based pediatric obesity program - preliminary results. Int J Exerc Sci. 2011;4(4):217-28.

83. Pelone F, Specchia ML, Veneziano MA, Capizzi S, Bucci S, Mancuso A, et al. Economic impact of childhood obesity on health systems: a systematic review. Obes Rev. 2012;13(5):431-40.

84. Robertson W, Fleming J, Kamal A, Hamborg T, Khan KA, Griffiths F, et al. Randomised controlled trial evaluating the effectiveness and cost-effectiveness of 'Families for Health', a family-based childhood obesity treatment intervention delivered in a community setting for ages 6 to 11 years. Health Technol Assess. 2017;21(1):1-180.

85. Hollingworth W, Hawkins J, Lawlor DA, Brown M, Marsh T, Kipping RR; Economic evaluation of lifestyle interventions to treat overweight or obesity in children. Int J Obes. 2012;36(4):559-566.

86. Hollinghurst S, Hunt LP, Banks J, Sharp DJ, Shield JP. Cost and effectiveness of treatment options for childhood obesity. Pediatr Obes. 2014;9(1):e26-34.

87. Wake M, Gold L, McCallum Z, Gerner B, Waters E. Economic evaluation of a primary care trial to reduce weight gain in overweight/obese children: the LEAP trial. Ambul Pediatr. 2008;8(5):336-341.

88. Soto-Gordoa M, Arrospide A, Hernández MM, Mora Amengual JM, Zabala AF, Larrañaga I, et al. Incorporating budget impact analysis in the implementation of complex interventions: a case of an integrated intervention for multimorbid patients within the CareWell study. Value in Health. 2017;20(1):100-06.

89. Burke LE, Wang J, Sevick MA. Self-monitoring in weight loss: a systematic review of the literature. J Am Diet Assoc. 2011;111(1):92-102.

90. Vine M, Hargreaves MB, Briefel RR, Orfield C. Expanding the role of primary care in the prevention and treatment of childhood obesity: a review of clinic- and community-based recommendations and interventions. J Obes. 2013.

91. Kay MC, Burroughs J, Askew S, Bennett GG, Armstrong S, Steinberg DM. Digital weight loss intervention for parents of children being treated for obesity: a prospective cohort feasibility trial. J Med Internet Res. 2018;20(12):e11093.

92. Antwi FA, Fazylova N, Garcon MC, Lopez L, Rubiano R, Slyer JT. Effectiveness of web-based programs on the reduction of childhood obesity in school-aged children: a systematic review. JBI Evid Synth. 2013;11(6):1-44.

93. Moore JB, Dilley JR, Singletary CR, Skelton JA, Miller Jr DP, Heboyan V, et al. A clinical trial to increase selfmonitoring of physical activity and eating behaviors among adolescents: protocol for the ImPACT feasibility study. JMIR Res Protoc. 2020;9(6):e18098.

94. Davison KK, Birch LL. Childhood overweight: a contextual model and recommendations for future research. Obes Rev. 2001;2(3):159-71.

95. Wolfenden L, Wyse R, Nichols M, Allender S, Millar L, McElduff P. A systematic review and meta-analysis of whole of community interventions to prevent excessive population weight gain. Prev Med. 2014;62:193-200.

\section{Figures}




\begin{tabular}{|c|c|c|}
\hline & iChoose & Family Connections \\
\hline$\rightleftarrows$ & $\begin{array}{l}6 \text { month intervention } \\
\text { (64 intended contact hours) }\end{array}$ & $\begin{array}{l}6 \text { month intervention } \\
\text { (5 intended contact hours) }\end{array}$ \\
\hline & $\begin{array}{l}\text { Guiding principles: } \\
\text { - Based on social learning theory and behavior } \\
\text { modification } \\
\text { - Family-based, non-dieting approach } \\
\text { Focuses on nutrition education, increasing } \\
\text { physioal activity and behavior modification } \\
\text { tochniques }\end{array}$ & $\begin{array}{l}\text { Guiding principles: } \\
\text { - Based on social-ecologic theory } \\
\text { Family-based, non-dieting approach } \\
\text { Focuses on improving the home environment, } \\
\text { parenting skills, role modeling, and positive } \\
\text { parental health behaviors related to healthful } \\
\text { eating, physical activity promotion, and weight } \\
\text { management }\end{array}$ \\
\hline & Parent/child dyads & Parent as change agent \\
\hline & $\begin{array}{l}12 \text { family classes (2 hours each; bi- } \\
\text { weekly; parent/child weigh-ins) }\end{array}$ & $\begin{array}{l}2 \text { parent classes ( } 90 \text { minutes each; } 1 \\
\text { week apart) }\end{array}$ \\
\hline & Clear communication workbook & Clear communication workbook \\
\hline & $\begin{array}{l}12 \text { IVR calls (10-20 minutes each; bi- } \\
\text { weekly): } \\
\text { - Taillored calls based on familly progress } \\
\text { towards goals } \\
\text {. } 5 \text { A's process of atsosement, advieing on new } \\
\text { program information, agreeing on new goals, } \\
\text { assisting with barriers, and arranging for } \\
\text { follow-up } \\
\text { Reinforces guiding principies } \\
\text {. Apply teach back and teach to goal } \\
\text { approaches to improve comprehension }\end{array}$ & $\begin{array}{l}\text { 10 IVR calls (5-15 minutes each; } 4 \\
\text { weekly, } 4 \text { bi-weekly, } 2 \text { monthly): } \\
\text { - Talilored calls based on family progress } \\
\text { towards gaals } \\
\text {. } 5 \text { A's process of atsessment, advising on new } \\
\text { program information, gareeing on new goals, } \\
\text { assisting with barriers, and arranging for } \\
\text { follow-up } \\
\text { - Reinforces guiding principles } \\
\text { - Trains parents to complete } 5 \mathrm{~A} \text { 's with their } \\
\text { family }\end{array}$ \\
\hline$\kappa$ & $\begin{array}{l}48 \text { family exercise sessions ( } 1 \text { hour; } 2 \\
\text { times per week) }\end{array}$ & \\
\hline 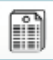 & 12 child newsletters (bi-weekly) & \\
\hline
\end{tabular}

\section{g(8) 3 Recruitment}

Eligible families: Children ages 8 12 with a BMI percentile $\geq 85^{\text {th }}$

Targeted referral system:

- Clinic partners identified potentially eligible children through a medical chart review

- Personalized letters from pediatrician were then mailed to all potential families

-Up to 6 contact attempts were made (combination of calls and tert messages)

-Eligible and interested families were scheduled for a baseline assessment

Open referral system: - Strategies: local newspapers and Facebook advertisements, flyers sent home through schools, Parent Advisory Team (PAT) and Parks \& Rec staff attended local health fairs and community events to help -Initial screening via telephone or in person and with a follow-up baseline health screening for those interested and eligible

Additions and Adaptations Cohort 3:

- Expanded criteria to also include children ages 5-8 with a BMI percentile $\geq 85^{\mathrm{m}}$

\section{Implementation}

Training:

- Consultee-centered approach that included knowledge development, role playing, self-assessment, goalsetting, and feedback

- 5 hour in-person Parks \& Rec staff training prior to cohort 1

- Teleconference training each week of family class implementation

Fidelity Measurement:

- Class fidelity evaluated using a

structured checklist customized for learning objectives

- Class fidelity objectively assessed by a researcher

- Physical activity class fidelity was self-reported by program staff on meeting goals for minutes of moderate to vigorous physical activity [iChoose only]

\section{Additions and Adaptations} Cohort 2 and 3:

Due to high fidelity in Cohort 1 and management of multiple program sites, Cohorts 2 and 3 included combination of objectively observed fidelity by researcher and self-rated fidelity by program staff [iChoose only]

\section{(idi) Engagement}

- Post card, calls, and texts reminders for data assessments

- Program welcome calls and/or texts from PAT members

- Regular class reminders via text message

- PAT attended classes to help facilitate family involvement

- Program staff and PAT provided a safety net for disengaged familie (e.g., attempted personal calls and/or texts if families missed more than two intended activities in a row)

- Non-monetary in-class incentives (e.g. t-shirts water bottles, physical activity props)

\section{Additions and Adaptations} Cohort 2 and 3 :

- Mini content overview videos texted to families who missed classes

Positive reinforcement and tips texted to parents from PAT members

- Retention strategies and goal setting by program staff - Enhanced use of Facebook [iChoose only]

Figure 1. Comparison of family-based childhood obesity (FBCO) treatment interventions and strategies for recruitment, implementation, and engagement and retention

\section{Figure 1}

Comparison of family-based childhood obesity (FBCO) treatment interventions and strategies for recruitment, implementation, and engagement and retention 


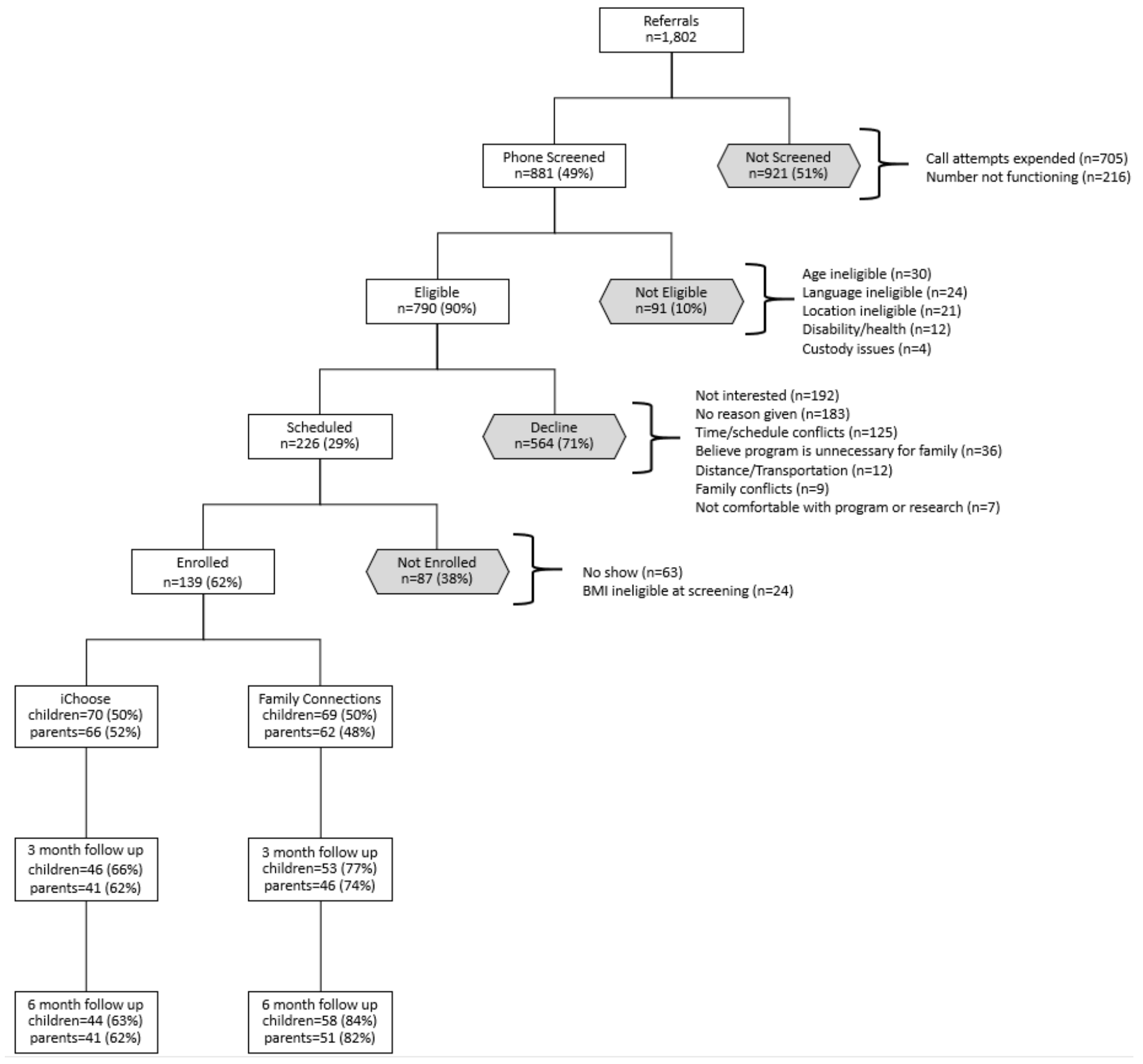

Figure 2

Consort flow diagram. Note: Families who responded to a contact and declined but asked to be contacted in the future, as well as those unable to be contacted but whose child remained age eligible, were contacted again for the subsequent cohort. As such, the 1,802 referrals represent 1,474 unique families. 


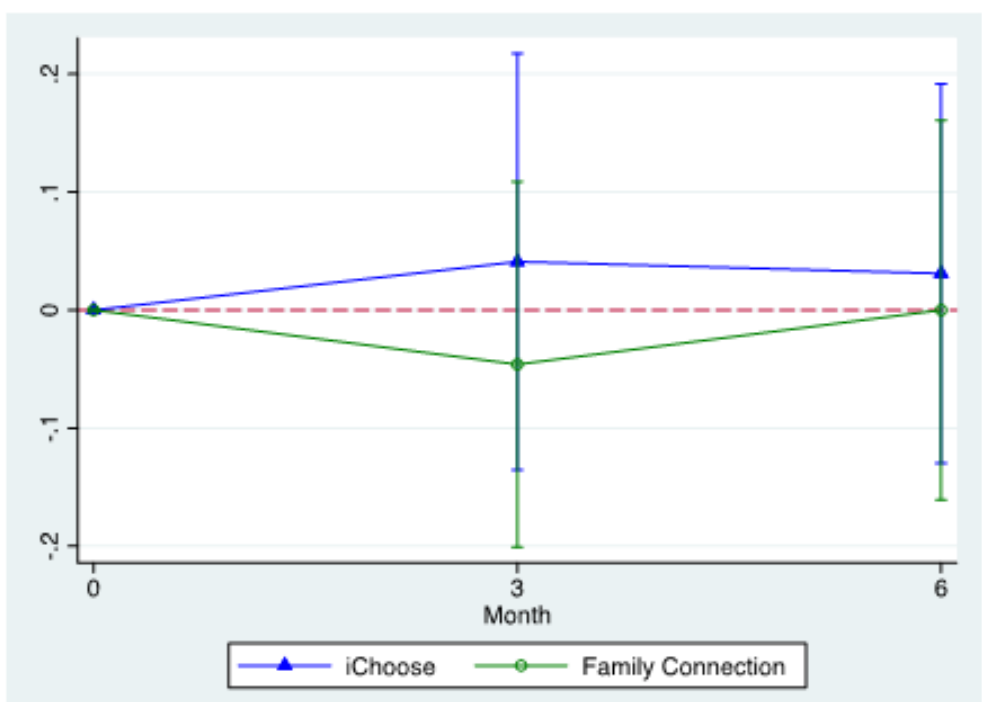

Figure 3a. Estimated children BMI z-score change overtime, by intervention condition (Mean and $95 \% \mathrm{CI}$ using Heckman approach)

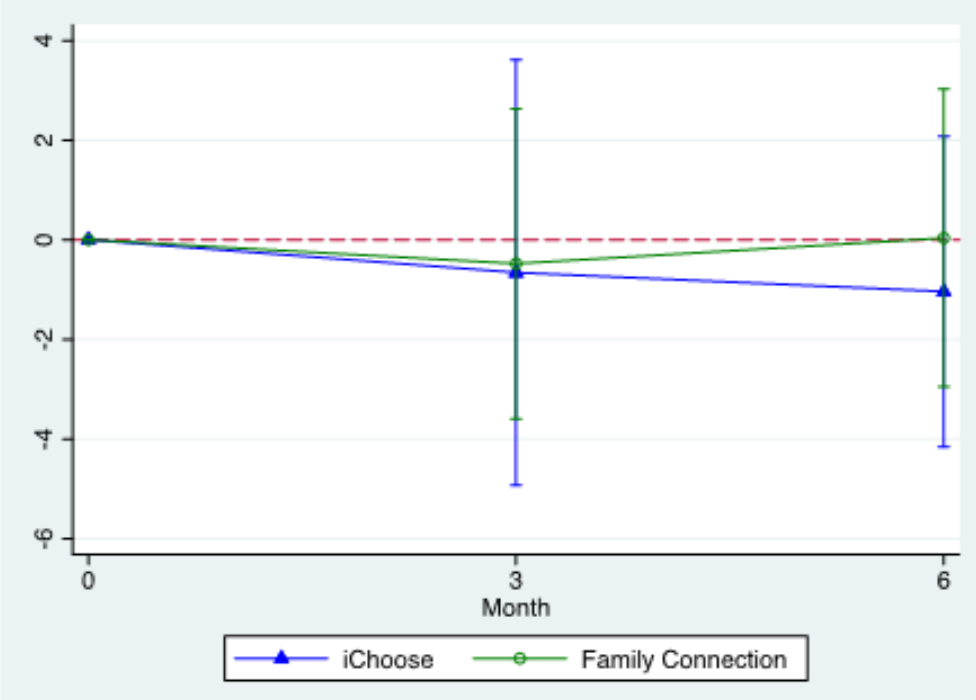

Figure 3b. Estimated parent BMI change overtime, by intervention condition (Mean and $95 \%$ CI using Heckman approach)

\section{Figure 3}

a. Estimated children BMI z-score change overtime, by intervention condition (Mean and 95\% $\mathrm{Cl}$ using Heckman approach). b. Estimated parent BMI change overtime, by intervention condition (Mean and 95\% $\mathrm{Cl}$ using Heckman approach) 


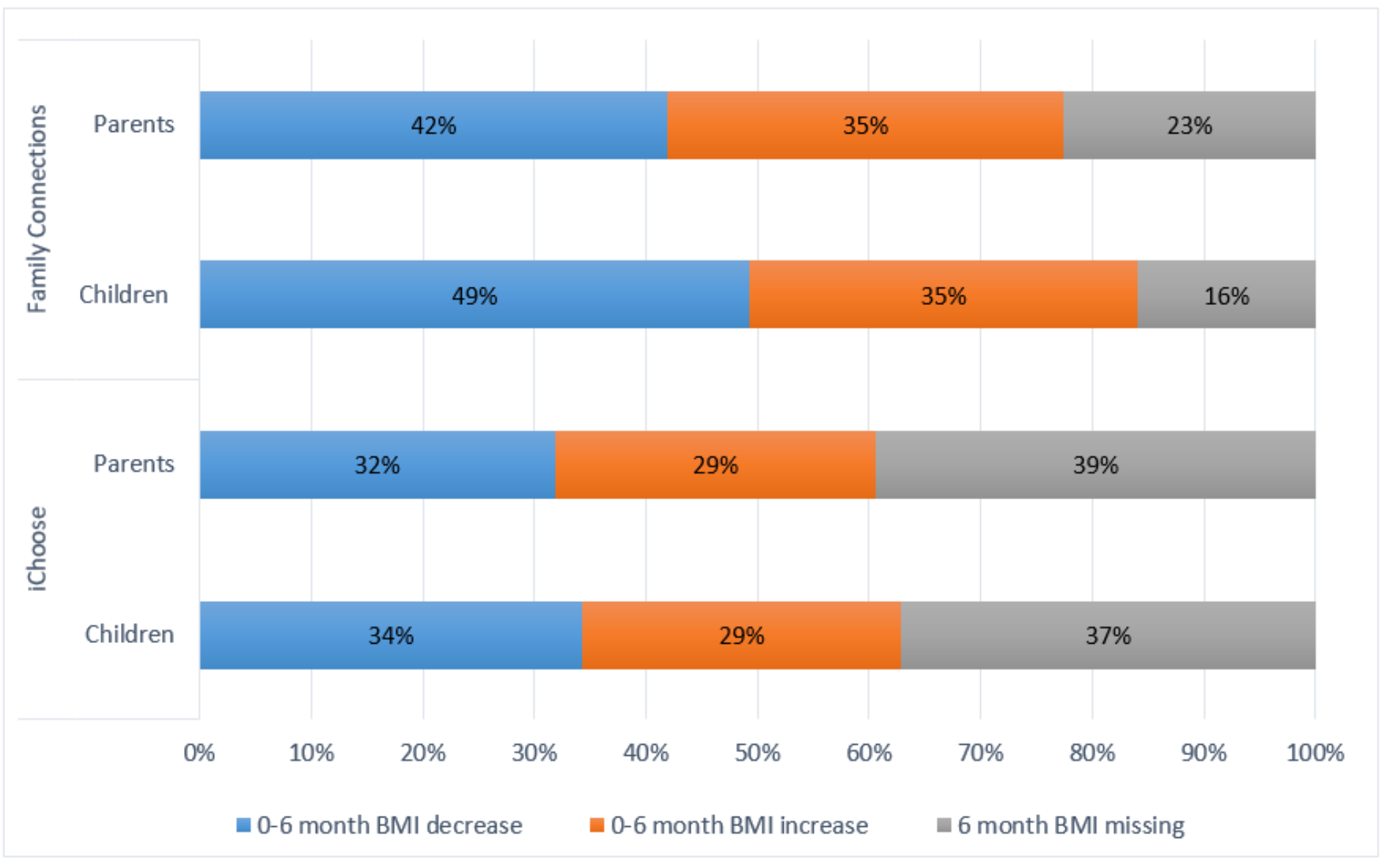

\section{Figure 4}

Proportion of children and parents, by intervention condition, with 0-6 month decrease, increase, or missing BMI data. Note: Children is change in BMI z-score, parent is change in BMI 


\begin{tabular}{|c|c|c|c|c|c|c|}
\hline & \multicolumn{3}{|l|}{ Choose } & \multicolumn{3}{|l|}{ Fanily Connections } \\
\hline & Description & Total & & Description & Total & \\
\hline \multicolumn{7}{|l|}{ Personnel costs } \\
\hline Initial staff training & $\begin{array}{l}\text { Initial } 5 \text { hour in-person trainirg } x 2 \\
\text { staff }\end{array}$ & $\$ 180$ & & Initial 5 hour in-person trainirg & $\$ 90$ & \\
\hline Weektystaff training & $\begin{array}{l}(20 \text { min bi-weekty video }+40 \\
\text { mintes bi-weelty meetirg) } \times 12 \\
\text { sessions } \times 2 \text { staff }\end{array}$ & $\$ 431$ & & $\begin{array}{l}(20 \text { min weekly video }+40 \text { mintes weekly } \\
\text { meetirg) } \times 2 \text { sessions }\end{array}$ & $\$ 36$ & \\
\hline Staffpreparation for qoup classes & 1 hour $\times 12$ sessions $\times 2$ staff & $\$ 431$ & & 1 hour $\mathrm{X} 2$ sessions & $\$ 36$ & \\
\hline Staff implementation for group classes & $\begin{array}{l}(2 \text { hours }+30 \text { min set-up }) \times 12 \\
\text { sessions } \times 2 \text { groups } \times 2 \text { staff }\end{array}$ & $\$ 2.156$ & & $\begin{array}{l}\text { (2 hours }+30 \text { min set-up) } \times 2 \text { sessions } \times 2 \\
\text { groups }\end{array}$ & $\$ 180$ & \\
\hline Staff preparation for physical actvity group classes & $15 \min x 36$ sessions & $\$ 162$ & & $\mathrm{NA}$ & SO & \\
\hline S taff implementation for plysical activity classes & $\begin{array}{l}(1 \text { hou }+15 \text { min set-up }) \times 36 \\
\text { sessions }\end{array}$ & $\$ 809$ & & NA & SO & \\
\hline Managirg participant engagement & 30 minutes $\times 24$ weelcs & $\$ 216$ & & 30 minutes $\mathrm{x} 10$ weeks & $\$ 90$ & \\
\hline Managing IVR & 15 min week $\times 24$ weeks & $\$ 108$ & & 15 min week $x 10$ weeks & $\$ 54$ & \\
\hline Newsletters & 1 hour $\times 12$ newsletters & $\$ 216$ & & $\mathrm{NA}$ & So & \\
\hline Personnd costs subtotal, without $P A T$ & & $\$ 4,708$ & & & $\$ 485$ & \\
\hline Parent Advsiory Teammeber & $\begin{array}{l}\text { \$200imonthly stipend per PAT } \\
\text { menber } x 4 \text { PAT menbers } x 6 \\
\text { months }\end{array}$ & $\$ 4,800$ & & $\begin{array}{l}\text { \$200 monfhly stipend per PAT menber } \times 2 \text { PAT } \\
\text { menbers } \times 6 \text { morths }\end{array}$ & $\$ 2.400$ & \\
\hline Personnel costs subtatal, with $P A T$ & & $\$ 9.508$ & & & $\$ 2.885$ & \\
\hline \multicolumn{7}{|l|}{ Other costs } \\
\hline Programmaterials (w orkbools \& supplies) & $\begin{array}{l}\text { Worksbooks, class supplies, and } \\
\text { mon-monetary class retention items }\end{array}$ & $\$ 3,559$ & & $\begin{array}{l}\text { Worksbooks, class supplies, and non-monetary } \\
\text { class retertion items }\end{array}$ & $\$ 1,049$ & \\
\hline IVR cost & $\$ 800$ per month $\times 6$ month & $\$ 4,800$ & & $\$ 800$ per month $x 8$ months & $\$ 6,400$ & \\
\hline Faclity costs for group classes & 12 famly sessions & $\$ 3,060$ & & 2 parent sessions & $\$ 510$ & \\
\hline Faclity costs for physical activity classes & 36 physical activity sessions & $\$ 1,836$ & & & & \\
\hline \multirow{2}{*}{ Other costs subtot al } & & $\$ 13,255$ & & & $\$ 7,959$ & \\
\hline & & $\begin{array}{l}\text { Per } \\
\text { cohort }\end{array}$ & $\begin{array}{l}\text { All3 } \\
\text { cohorts }\end{array}$ & & $\begin{array}{l}\text { Per } \\
\text { cohort }\end{array}$ & $\begin{array}{l}\text { All3 } \\
\text { colhorts }\end{array}$ \\
\hline TOTAL per colort; with PAT, with facility & & $\$ 22,763$ & $\$ 68,289$ & & $\$ 10,844^{7}$ & $\$ 32,533$ \\
\hline TOTAL per colnort; without PAT, with facility & & $\$ 17.963^{\prime}$ & $\$ 53.889$ & & $\$ 8,444^{\prime}$ & $\$ 25.333$ \\
\hline T OTAL per colnort, without PAT, withou facility & & $\$ 13.067^{\prime}$ & $\$ 39.201$ & & $\$ 7934^{\circ}$ & $\$ 23.803$ \\
\hline
\end{tabular}

\section{Figure 5. Estimated program implementation costs by treatment condition}

Notes

- All estimated cost rounded to nearest whole dollar

- The average hourly rate plus fringe benefits of local bachelors-level trained Parks \& Rec program staff (i.e., $\$ 17.97 /$ hour) was used to estimate personnel costs for both iChoose and Family Connections.

- Two available times were available for each in-person family/parent class session (mid-morning and early afternoon) for both $i$ Choose and Family Connections, but only one time was available for $i$ Choose physical activity sessions.

- $\quad i$ Choose implementation requires two program staff to oversee parent and child groups at the 12 bi-weekly family classes, yet only one staff to oversee physical activity sessions and other participant management.

- Family Connections implementation requires one staff member at the two parent classes and to oversee participant management.

\section{Figure 5}

Estimated program implementation costs by treatment condition 


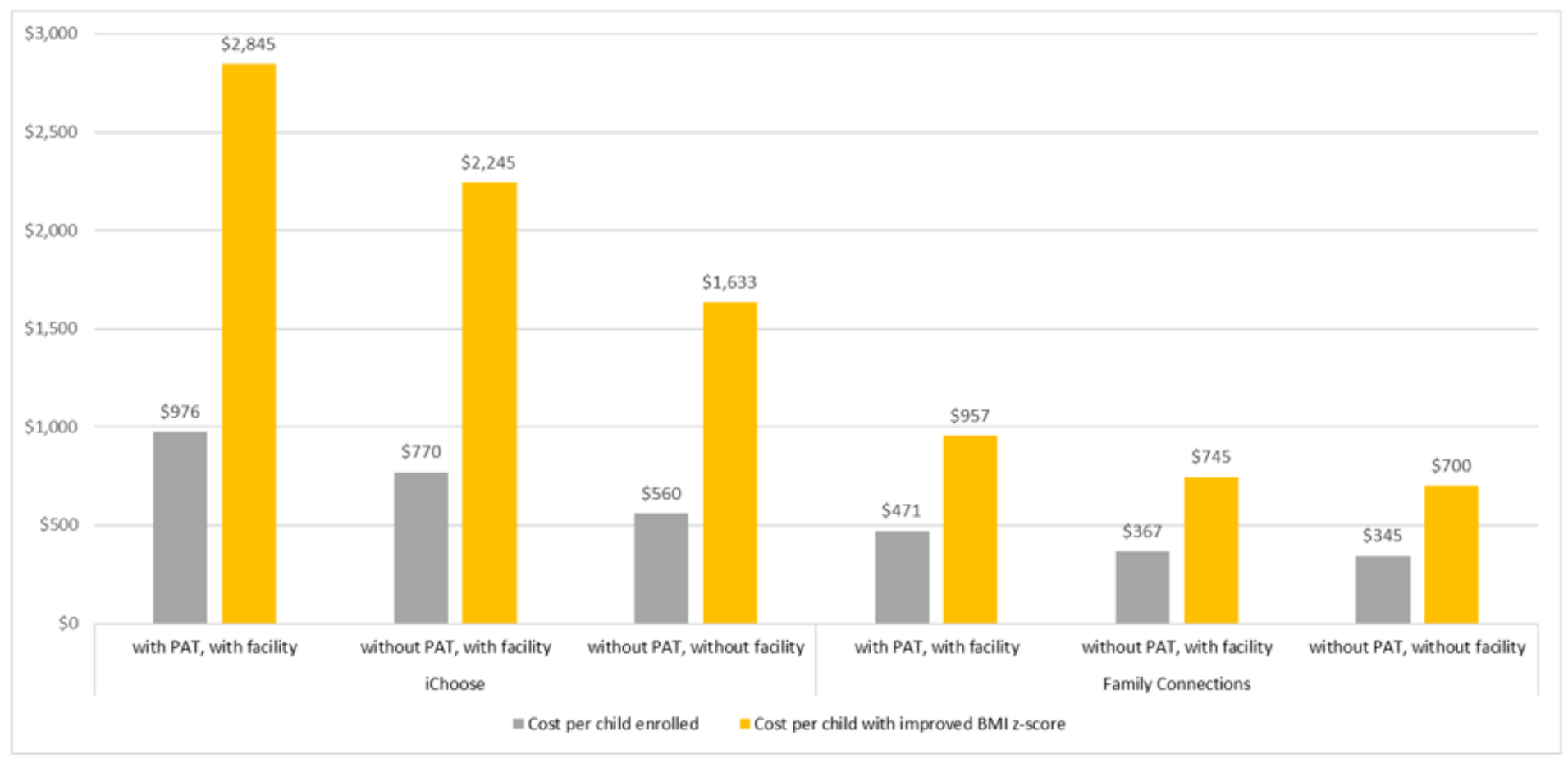

Figure 6

Program implementation cost per enrolled child and per enrolled child with improved 0-6 month BMI outcomes, by intervention condition. Note: PAT = Parent Advisory Team

\section{Supplementary Files}

This is a list of supplementary files associated with this preprint. Click to download.

- CONSORT2010Checklist1.doc 